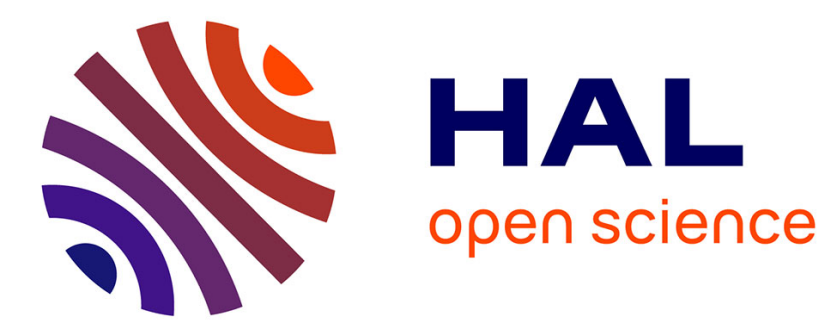

\title{
Effect of control parameters on biogas production during the anaerobic digestion of protein-rich substrates
}

\author{
Z. Khedim, Boumédiène Benyahia, Brahim Cherki, Tewfik Sari, Jérôme \\ Harmand
}

\section{- To cite this version:}

Z. Khedim, Boumédiène Benyahia, Brahim Cherki, Tewfik Sari, Jérôme Harmand. Effect of control parameters on biogas production during the anaerobic digestion of protein-rich substrates. Applied Mathematical Modelling, 2018, 61, pp.351-376. 10.1016/j.apm.2018.04.020 . hal-02608027

\section{HAL Id: hal-02608027 \\ https://hal.inrae.fr/hal-02608027}

Submitted on 26 May 2020

HAL is a multi-disciplinary open access archive for the deposit and dissemination of scientific research documents, whether they are published or not. The documents may come from teaching and research institutions in France or abroad, or from public or private research centers.
L'archive ouverte pluridisciplinaire HAL, est destinée au dépôt et à la diffusion de documents scientifiques de niveau recherche, publiés ou non, émanant des établissements d'enseignement et de recherche français ou étrangers, des laboratoires publics ou privés.

$$
\text { Copyright }
$$




\section{Accepted Manuscript}

Effect of control parameters on biogas production during the anaerobic digestion of protein-rich substrates

Zeyneb Khedim, Boumediène Benyahia, Brahim Cherki, Tewfik Sari, Jérôme Harmand

PII:

DOI:

Reference:

To appear in:

Received date:

Revised date:

Accepted date:
S0307-904X(18)30205-1

10.1016/j.apm.2018.04.020

APM 12259

Applied Mathematical Modelling

8 September 2017

13 February 2018

25 April 2018

Please cite this article as: Zeyneb Khedim, Boumediène Benyahia, Brahim Cherki, Tewfik Sari, Jérôme Harmand, Effect of control parameters on biogas production during the anaerobic digestion of protein-rich substrates, Applied Mathematical Modelling (2018), doi: 10.1016/j.apm.2018.04.020

This is a PDF file of an unedited manuscript that has been accepted for publication. As a service to our customers we are providing this early version of the manuscript. The manuscript will undergo copyediting, typesetting, and review of the resulting proof before it is published in its final form. Please note that during the production process errors may be discovered which could affect the content, and all legal disclaimers that apply to the journal pertain. 


\section{Highlights}

- A model of Anaerobic Digestion of protein-rich Microalgae (MAD) was analyzed

- An insight on the process behavior as a function of control parameters was provided

- Operating Diagram defined the ideal conditions to optimize biogas yield and ammonia toxicity

- Application of MAD model is limited in acidic environments

- Qualitative-quantitative properties of ADM1m model were derived from those of MAD

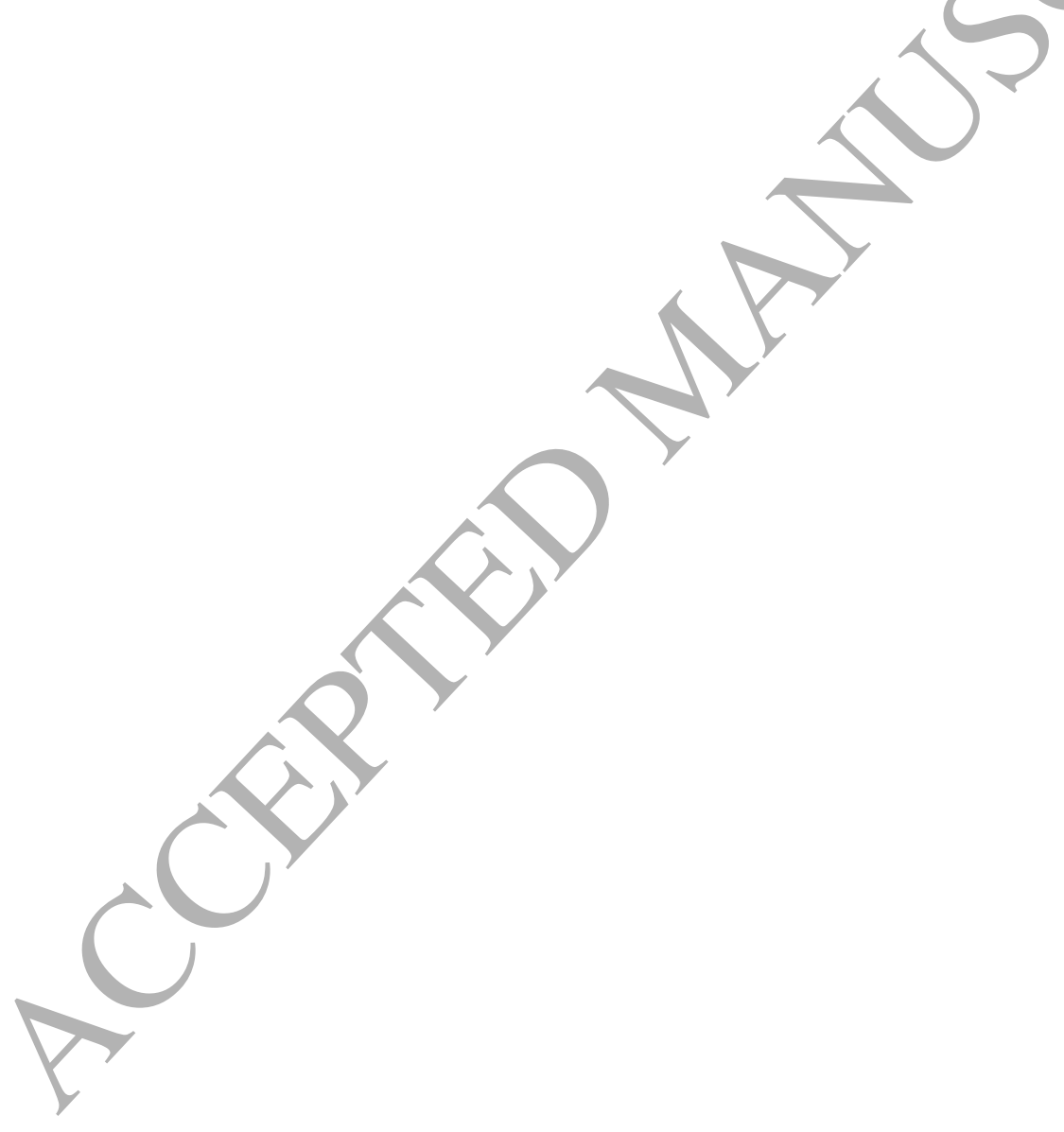




\title{
Effect of control parameters on biogas production during the anaerobic digestion of protein-rich substrates
}

\author{
Zeyneb Khedim ${ }^{\mathrm{a}, *}$, Boumediène Benyahia ${ }^{\mathrm{a}}$, Brahim Cherki ${ }^{\mathrm{a}}$, Tewfik Sari ${ }^{\mathrm{b}}$, Jérôme \\ Harmand $^{\mathrm{c}}$ \\ ${ }^{a}$ Laboratoire d'Automatique de Tlemcen, Université de Tlemcen. BP 230 Tlemcen 13000, Algeria \\ ${ }^{b}$ Irstea, UMR ITAP, Univ Montpellier, 34196, Montpellier, France \\ ${ }^{c}$ LBE, INRA, Univ Montpellier, 11100, Narbonne, France
}

\begin{abstract}
This paper deals with the theoretical study of the anaerobic digestion of proteinrich substrates. Since the success of such digestion raises the issue of the possible release of ammonia, we have carried out a mathematical analysis of an Anaerobic Digestion model specifically developed for the treatment of Microalgae, termed MAD (Microalgae Anaerobic Digestion) in the literature. Our aim is to investigate the qualitative properties of the system via the calculation of its equilibria, their conditions of existence as well as their stability according to the operating parameters values. Simulation results highlight the key parameters acting on model behavior. In particular, it is shown that the control parameters can greatly affect biogas yield and, thus, process performance. The optimal conditions for process operation are then identified. To emphasize the effect of $\mathrm{pH}$ on biological reactions, we plot the operating diagram of the MAD model for different fixed $\mathrm{pH}$ values. We demonstrate that a rising $\mathrm{pH}$ favors the formation of the free ammonia, leading ultimately to methanogen inhibition. Surprisingly, our study highlights the insensitivity of the model to acidic environments, thus limiting its potential applications at low pH. Finally, we investigate the qualitative and quantitative properties of a modified ADM1 (Anaerobic Digestion Model $\mathrm{n}^{\circ} 1$ ) model in the light of those of the MAD model.
\end{abstract}

Keywords: Anaerobic Digestion, equilibria, model, Microalgae, operating diagram, stability analysis

*Corresponding author

Email addresses: zeyneb.khedim@univ-tlemcen.dz (Zeyneb Khedim),

b.benyahia.ut1@gmail.com (Boumediène Benyahia), b.cherki@gmail.com (Brahim Cherki),

tewfik.sari@irstea.fr (Tewfik Sari), jerome.harmand@inra.fr (Jérôme Harmand) 


\section{Introduction}

Anaerobic Digestion (AD) is a biological process, which reduces organic pollution and produces renewable energy (biogas). This kind of bioprocess is regarded as an alternative energy source to fossil fuel.

In order to maximize its efficiency, anaerobic digestion is now widely used at fullscale to degrade various organic feedstocks. Special Attention has been paid to the fermentation of protein-rich substrates, in view of their outstanding yield of biogas and fertilizers [1], [2].

However, despite its energetic benefit, the fermentation of such substrates leads to high concentrations of nitrogen in the reaction medium derived from the breakdown of protein. Nitrogen is converted into ammonium $\left(\mathrm{NH}_{4}^{+}\right)$and free ammonia $\mathrm{NH}_{3}$, which is toxic for bacterial communities at high concentrations. Ammonia toxicity can cause serious problems of instability leading to dramatic damage of the process and even its failure.

Environmental factors such as temperature, $\mathrm{pH}$, bacterial acclimation, can have a significant effect on such phenomena. Furthermore, control parameters as the dilution rate or the feed concentration at inlet might significantly influence the whole process performances [3].

Many researches were established to assess operating conditions impact on nitrogen inhibition. In this context, ammonia influence on AD has been evaluated at a mesophilic temperature [4]. Its threshold level for inhibition has been ascertained for the anaerobic digestion of saline wastewater [5], while the impact of its concentration and of Hydraulic Retention Time (HRT) has been studied in [6].

To deal with such an inhibition in practice, alternative solutions have been also suggested either by: i) the addition of certain elements to the feed substrate (codigestion of meat and kitchen waste to extend the $\mathrm{C}: \mathrm{N}$ ratio [2] and adding trace elements throughout the Anaerobic Digestion of food wastes [7]); or by ii) keeping the factors limiting ammonia inhibition at their optimum values in testing the impact of Organic Loading Rate (OLR) and Hydraulic Retention Time (HRT) on the AD of animal byproducts [8], and investigate $\mathrm{pH}$ impact on the $\mathrm{AD}$ of kitchen waste [9].

In addition, some experimental applications has been focused on the optimization of protein-rich substrates digestion. The OLR was optimized under different temperatures throughout the digestion of slaughterhouse waste [10]. The useful agents for maximizing biogas generation from the $\mathrm{AD}$ of slaughterhouse by-products (blood, meat, ribs, fat, raw waste) were studied in [11], while the efficacy of co-digestion of gelatin and turkey was addressed in [12]. 
Elsewhere, other studies have modeled the digestion of protein-rich substrates such as the models proposed by Batstone et al. [13] and Lokshina et al.[14], developed to describe the AD of slaughterhouse wastewater. Besides, Angelidaki et al. were interested in the validation of an existing model of AD using experimental data of the co-digestion of manure and protein-rich wastewater [15].

In [16], the AD of chlorella vulgaris microalgae was considered, in terms of composition, as a source of proteins. A model for Microalgae AD (hereafter denoted as the "MAD model") were proposed. It was validated on experimental data and compared to a modified ADM1 model specifically adapted to the digestion of microalgae [17].

Most previous studies used experimental results of various substrates digestion to derive ideal values for operating parameters, while our present contribution is to emphasize [17] in simulation the influence of these parameters on the qualitative behavior of the process. To do so, we investigate the properties of the MAD model and we compare them to those of ADM1

In this paper, we focus on the mathematical study of the MAD model in order to identify ideal conditions of the process operation. An exhaustive analysis of its equilibria, their stability and their bifurcation properties was established. Subsequently, process performance was evaluated in terms of biogas production in order to define the optimal ranges of $\mathrm{pH}$, dilution rate $(D)$, organic load $\left(S_{\text {in }}\right)$ and inorganic nitrogen concentration $\left(N_{i n}\right)$, which ensuring maximum yield from fermentation. Based on the present study, we show how control parameters affect system performance. In particular, the favorable conditions for ideal biogas production have been stressed. The paper is organized as follows: first, the MAD model is presented and its equilibria are calculated. Stability of equilibria is evaluated with respect of control parameters changes. Thereafter, the Operating Diagrams (OD) of the system are presented under different degrees of acidity to investigate the effect of $\mathrm{pH}$ on the anaerobic digestion. In addition, the process performance is assessed by following the evolution of biogas production. Simulation results highlight the effect of control parameters on the digestion and validate the improved theoretical analysis. Finally, discussions and general conclusions are drawn.

\section{Mathematical analysis of Microalgae Anaerobic Digestion model}

\subsection{Presentation of MAD model}

We consider here the three reaction-two step model of Microalgae Anaerobic Digestion (MAD) proposed by Mairet et al. [16]. The model involves four sub- 
strates ( $S_{1}$ sugars/lipids, $S_{2}$ proteins, $S_{3} \mathrm{VFAs}^{1}$ and $S_{I}$ inerts), and three bacterial groups ( $X_{1}$ sugar/lipid-degrading bacteria, $X_{2}$ protein-degrading bacteria and $X_{3}$ VFA-degrading methanogens), (see Figure 1). Mathematical equations of the MAD model are given by:

$$
\begin{aligned}
\dot{S}_{1} & =D\left(\beta_{1} S_{i n}-S_{1}\right)-\alpha_{1} \mu_{1} X_{1} \\
\dot{S}_{2} & =D\left(\beta_{2} S_{i n}-S_{2}\right)-\alpha_{5} \mu_{2} X_{2} \\
\dot{S}_{3} & =-D S_{3}+\alpha_{3} \mu_{1} X_{1}+\alpha_{6} \mu_{2} X_{2}-\alpha_{9} \mu_{3} X_{3} \\
\dot{X}_{1} & =\left(\mu_{1}-D\right) X_{1} \\
\dot{X}_{2} & =\left(\mu_{2}-D\right) X_{2} \\
\dot{X}_{3} & =\left(\mu_{3}-D\right) X_{3} \\
\dot{N} & \left.=D\left(N_{\text {in }}-N\right)-\alpha_{2} \mu_{1} X_{1}+\alpha_{7} \mu_{2} X_{2}-\alpha_{10} \mu_{3} X_{3}\right) \\
\dot{C} & =D\left(C_{i n}-C\right)+\alpha_{4} \mu_{1} X_{1}+\alpha_{8} \mu_{2} X_{2}+\alpha_{12} \mu_{3} X_{3}-\rho_{C O_{2}} \\
\dot{P}_{C O_{2}} & =-P_{C O_{2}} \frac{q_{\text {gas }}}{V_{\text {gas }}}+\rho_{C O_{2}} \frac{V_{\text {liq }} R T_{o p}}{V_{\text {gas }}} \\
\dot{P}_{C H_{4}} & =-P_{C H_{4}} \frac{q_{\text {gas }}}{V_{\text {gas }}}+\rho_{C H_{4}} \frac{V_{\text {liq }} R T_{\text {op }}}{V_{\text {gas }}} \\
\dot{z} & =D\left(z_{\text {in }}-z\right) \\
\dot{S}_{I} & =D\left(\beta_{I} S_{\text {in }}-S_{I}\right)
\end{aligned}
$$

where $D$ is the dilution rate, $\alpha_{i}(i=1, \ldots, 12)$ are the stoichiometric parameters, $\beta_{i}(i=$ $1,2, I)$ are the fractionation coefficients, $S_{i n}, N_{i n}, C_{i n}$ and $z_{i n}$ denote, respectively, inlet concentrations of the organic matter, the inorganic nitrogen $N$, the inorganic carbon $C$ and the alkalinity z.

$P_{\mathrm{CO}_{2}}$ and $P_{\mathrm{CH}_{4}}$ are the partial pressures of the $\mathrm{CO}_{2}$ and $\mathrm{CH}_{4}$ while $\rho_{\mathrm{CO}_{2}}$ and $\rho_{\mathrm{CH}_{4}}$ are their liquid-gas transfer rates defined as:

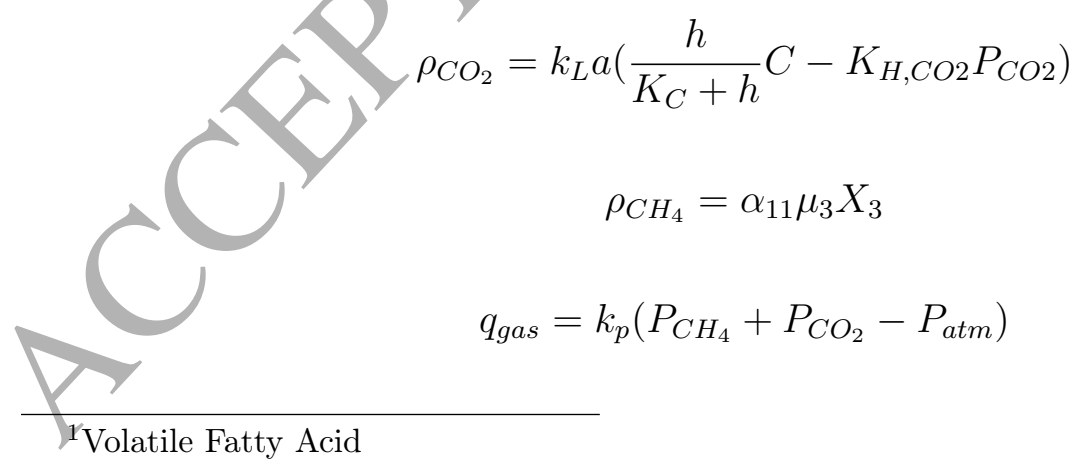


$V_{\text {liq }}, V_{\text {gas }}, R, k_{L} a, T_{o p}, P_{a t m}, q_{g a s}, k_{p}, h$ denote, respectively, the liquid volume, gas volume, gas law constant, physico-chemical coefficient, temperature, atmospheric pressure, gas flow, pipe resistance coefficient and the concentration of the hydrogen/hydroxide ions. $K_{C}$ and $K_{\mathrm{H}, \mathrm{CO} 2}$ are, respectively, chemical constants of dissociation and of volatility (Henry's law).

The kinetics $\mu_{1}$ and $\mu_{2}$ are ratio-dependent functions: they depend on $S_{i} / X_{i}(i=$ 1,2). Such functions are represented by the Contois model:

$$
\mu_{i}\left(S_{i}, X_{i}\right)=\bar{\mu}_{i} \frac{S_{i}}{\left(S_{i}+K_{S i} X_{i}\right)}, i=(1,2)
$$

where $\bar{\mu}_{i}(i=1,2)$ are the maximum specific growth rates of the bacteria $X_{i}, K_{S i}$ is the Contois half saturation constants.

The kinetic $\mu_{3}$ depends on both $S_{3}$ and $\mathrm{NH}_{3}$. It is modeled by the following function:

$$
\mu_{3}\left(S_{3}, N H_{3}\right)=\bar{\mu}_{3} \frac{S_{3}}{S_{3}+K_{S_{3}}+\frac{S_{3}^{2}}{K_{I 3}}} \frac{K_{I, n h_{3}}}{K_{I, n h_{3}}+N H_{3}}
$$

with $\bar{\mu}_{3}$ the maximum specific growth rate of the bacteria $X_{3}, K_{S 3}$ is the Haldane half saturation constant, $K_{I 3}$ is the Haldane inhibition constant and $K_{I, n h_{3}}$ is the ammonia inhibition constant.

The association or dissociation of total inorganic nitrogen $N$ with hydrogen/hydroxide ions determine the formation of ammonia $\mathrm{NH}_{3}$ and ammonium $\mathrm{NH}_{4}^{+}$. At equilibrium, this physico-chemical process is characterized by a dissociation constant $K_{N}$ defined as: $K_{N}=h\left[N H_{3}\right] /\left[N H_{4}^{+}\right]$. Thus, $\mu_{3}$ can be written as:

$$
\mu_{3}\left(S_{3}, N\right)=\bar{\mu}_{3} \frac{S_{3}}{S_{3}+K_{S_{3}}+\frac{S_{3}^{2}}{K_{I 3}}} \frac{K_{I, n h_{3}}}{K_{I, n h_{3}}+\lambda N}, \quad \lambda=\frac{K_{N}}{K_{N}+h}
$$

In view of low values of HRT adopted in the experiments of Mairet et al. [16], bacterial decay rate was not taken into account in the model's equations.

Hypothesis. $p H=-\log _{10}[h]$ constant, which implies that $\lambda$ is constant.

For simplicity, we overlook in the mathematical analysis the equations (8), (9) and (10), which are model outputs. The equations (11) and (12) are also ignored due to the assumption that the $\mathrm{pH}$ is constant.

Hence, we consider the reduced model (1)-(7) and we denote $\xi=\left[S_{1}, S_{2}, S_{3}, X_{1}\right.$, $\left.X_{2}, X_{3}, N\right]^{T}$ the state vector. 


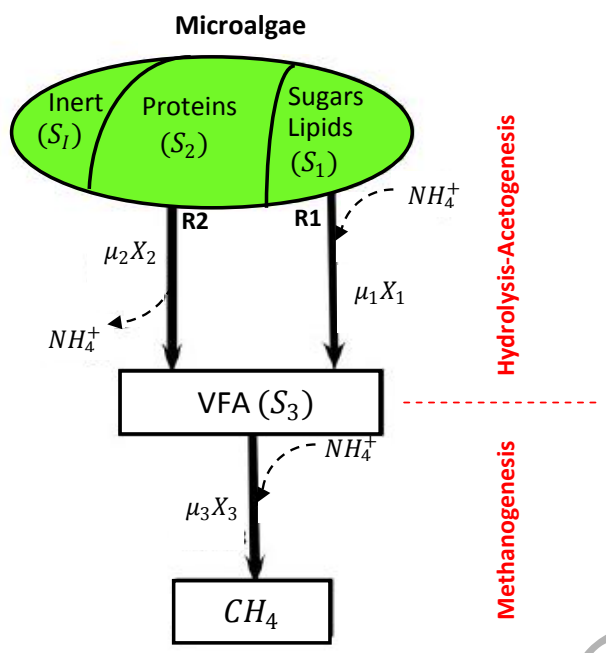

Figure 1: Reaction stages considered in the MAD model [16].

\subsection{Equilibria of the model}

The equilibria of the reduced MAD model are given by the solutions of the following system of algebraic equations:

$$
\left\{\begin{array}{l}
0=D\left(\beta_{1} S_{i n}-S_{1}\right)-\alpha_{1} \mu_{1} X_{1} \\
0=D\left(\beta_{2} S_{i n}-S_{2}\right)-\alpha_{5} \mu_{2} X_{2} \\
0=-D S_{3}+\alpha_{3} \mu_{1} X_{1}+\alpha_{6} \mu_{2} X_{2}-\alpha_{9} \mu_{3} X_{3} \\
0=\left(\mu_{1}-D\right) X_{1} \\
0=\left(\mu_{2}-D\right) X_{2} \\
0=\left(\mu_{3}-D\right) X_{3} \\
0 \neq D\left(N_{i n}-N\right)-\alpha_{2} \mu_{1} X_{1}+\alpha_{7} \mu_{2} X_{2}-\alpha_{10} \mu_{3} X_{3}
\end{array}\right.
$$

Eight cases are considered, which are reported in Table 1.

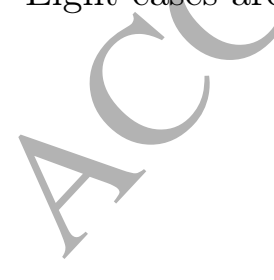


Table 1: Different cases for equilibria

\begin{tabular}{lllll}
\multicolumn{5}{c}{ Table 1: Different cases for equilibria } \\
\cline { 2 - 5 } & Case1 & $X_{1}=0$ & $X_{2}=0$ & $X_{3}=0$ \\
Case2 & $X_{1}=0$ & $\mu_{2}\left(S_{2}, X_{2}\right)=D$ & $X_{3}=0$ \\
Case3 & $X_{1}=0$ & $\mu_{2}\left(S_{2}, X_{2}\right)=D$ & $\mu_{3}\left(S_{3}, N\right)=D$ \\
calculation. & Case4 & $\mu_{1}\left(S_{1}, X_{1}\right)=D$ & $X_{2}=0$ & $X_{3}=0$ \\
& Case5 & $\mu_{1}\left(S_{1}, X_{1}\right)=D$ & $X_{2}=0$ & $\mu_{3}\left(S_{3}, N\right)=D$ \\
Case6 & $\mu_{1}\left(S_{1}, X_{1}\right)=D$ & $\mu_{2}\left(S_{2}, X_{2}\right)=D$ & $X_{3}=0$ \\
Case7 & $\mu_{1}\left(S_{1}, X_{1}\right)=D$ & $\mu_{2}\left(S_{2}, X_{2}\right)=D$ & $\mu_{3}\left(S_{3}, N\right)=D$ \\
Case8 & $X_{1}=0$ & $X_{2}=0$ & $\mu_{3}\left(S_{3}, N\right)=D$ \\
\hline
\end{tabular}

Due to the absence of the inlet concentration $S_{3 i n}$ of Volatile Fatty Acid in the model, case 8 is biologically impossible and thus it will be not taken into account. Hence, the equations $(19 \mathrm{a}, 19 \mathrm{~d}),(19 \mathrm{~b}, 19 \mathrm{e}),(19 \mathrm{c}, 19 \mathrm{f}$ and 19g) can be solved considering the following three subsystems of equations:

$$
\begin{gathered}
\begin{cases}\bar{\mu}_{1} \frac{S_{1}}{S_{1}+K_{S_{1}} X_{1}} & =\mathrm{D} \\
S_{1}+\alpha_{1} X_{1} & =\beta_{1} S_{i n}\end{cases} \\
\left\{\begin{array}{l}
\bar{\mu}_{2} \frac{S_{2}}{S_{2}+K_{S_{2}} X_{2}}=\mathrm{D} \\
S_{2}+\alpha_{5} X_{2}
\end{array}=\beta_{2} S_{i n}\right. \\
\left\{\begin{array}{l}
-S_{3}+\alpha_{6} X_{2}+\alpha_{3} X_{1}-\alpha_{9} X_{3}=0 \\
N_{i n}-N-\alpha_{2} X_{1}+\alpha_{7} X_{2}-\alpha_{10} X_{3}=0 \\
\bar{\mu}_{3} \frac{S_{3}}{S_{3}+K_{S_{3}}+\frac{S_{3}{ }^{2}}{K_{I 3}}} \frac{K_{I, n h_{3}}}{K_{I, n h_{3}}+\lambda N}=D
\end{array}\right.
\end{gathered}
$$

From the equation (20a), we have:

$$
S_{1}^{*}=\frac{D K_{S_{1}} X_{1}^{*}}{\bar{\mu}_{1}-D}
$$

which is non-negative if, and only if, $\bar{\mu}_{1}-D>0$.

By combining equations (20a) and (20b), we have:

$$
X_{1}^{*}=\frac{\beta_{1} S_{i n}}{\frac{D K_{S_{1}}}{\bar{\mu}_{1}-D}+\alpha_{1}}
$$


Combining this expression with (23), we obtain:

$$
S_{1}^{*}=\beta_{1} S_{i n} \frac{D K_{S_{1}}}{D K_{S_{1}}+\alpha_{1}\left(\bar{\mu}_{1}-D\right)}
$$

Thus, if $S_{1}^{*}$ exists i.e. if $D<\bar{\mu}_{1}$, then $S_{1}^{*}$ is necessarily smaller than $\beta_{1} S_{i n}$. From equation (21a) we calculate:

$$
S_{2}^{*}=\frac{D K_{S_{2}} X_{2}^{*}}{\bar{\mu}_{2}-D}
$$

which is non-negative if, and only if, $\bar{\mu}_{2}-D>0$.

Combining equations (21a) and (21b) we have:

$$
X_{2}^{*}=\frac{\beta_{2} S_{i n}}{\frac{D K_{S_{2}}}{\bar{\mu}_{2}-D}+\alpha_{5}}
$$

Combining the last expression with (26), we obtain:

$$
S_{2}^{*}=\beta_{2} S_{i n} \frac{D K_{S_{2}}}{D K_{S_{2}}+\alpha_{5}\left(\bar{\mu}_{2}-D\right)}
$$

Thus, if $S_{2}^{*}$ exists i.e. if $D<\bar{\mu}_{2}$, then $S_{2}^{*}$ is necessarily smaller than $\beta_{2} S_{i n}$.

From (22a), we get:

$$
X_{3}^{*}=\frac{1}{\alpha_{9}}\left(S_{3 i n}^{*}-S_{3}^{*}\right)
$$

Equation (22b) gives:

with

$$
N^{*}=N_{i n}^{*}-\alpha_{10} X_{3}^{*}
$$

$S_{3 i n}^{*}= \begin{cases}S_{3 i n, 1}^{*}=\alpha_{3} X_{1}^{*} & \text { if only the reaction1 (R1) occurs (Figure 1) } \\ S_{3 i n, 2}^{*}=\alpha_{6} X_{2}^{*} & \text { if only the reaction2 (R2) occurs (Figure 1) } \\ S_{3 i n, t o t a l}^{*}=\alpha_{3} X_{1}^{*}+\alpha_{6} X_{2}^{*} \quad \text { if both reactions (R1) and (R2) occur (Figure 1) }\end{cases}$

$N_{i n}^{*}=\left\{\begin{array}{l}N_{i n, 1}^{*}=N_{i n}-\alpha_{2} X_{1}^{*} \quad \text { if only the reaction1 (R1) occurs (Figure 1) } \\ N_{i n, 2}^{*}=N_{i n}+\alpha_{7} X_{2}^{*} \quad \text { if the only reaction2 (R2) occurs (Figure 1) } \\ N_{\text {in,total }}^{*}=N_{i n}-\alpha_{2} X_{1}^{*}+\alpha_{7} X_{2}^{*} \quad \text { if both reactions (R1) and (R2) occur (Figure 1) }\end{array}\right.$

Figure 2 illustrates the cascade structure of the system leading to different concentrations of $S_{3 i n}^{*}$ and $N_{i n}^{*}$. 


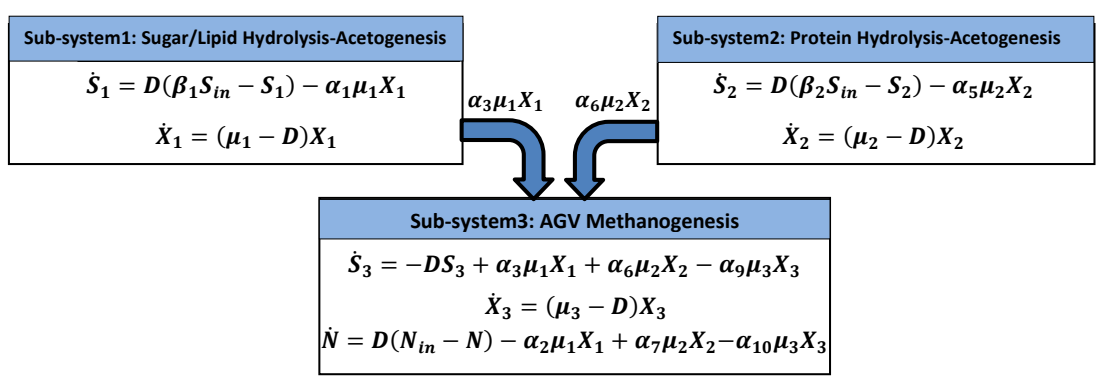

Figure 2: The structure of the system.

From (29) and (30), we deduce that

$$
N^{*}=A+\frac{\alpha_{10}}{\alpha_{9}} S_{3}^{*}, \quad \text { where } A:=N_{i n}^{*}-\frac{\alpha_{10}}{\alpha_{9}} S_{3 i n}^{*}
$$

Replacing this expression in (22c), we find that $S_{3}^{*}$ is the solution of the following equation:

$$
\bar{\mu}_{3} \frac{S_{3}}{S_{3}+K_{S_{3}}+\frac{S_{3}{ }^{2}}{K_{I 3}}}=D \frac{K_{I, n h_{3}}+\lambda\left(A+\frac{\alpha_{10}}{\alpha_{9}} S_{3}\right)}{K_{I, n h_{3}}}
$$

Thus, $S_{3}$ is the intersection of a Haldane function $f\left(S_{3}\right)$ with a rising line $g\left(S_{3}\right)$ defined as:

$$
\begin{gathered}
f\left(S_{3}\right)=\bar{\mu}_{3} \frac{y^{\prime} S_{3}}{S_{3}+K_{S_{3}}+\frac{S_{3}{ }^{2}}{K_{I 3}}} \\
g\left(S_{3}\right)=D \frac{K_{I, n h_{3}}+\lambda\left(A+\frac{\alpha_{10}}{\alpha_{9}} S_{3}\right)}{K_{I, n h_{3}}}
\end{gathered}
$$

Hence, we can obtain 2,1 or 0 intersection(s). There are, thus, at most only two positive equilibria. This is schematically represented in Figure 3.
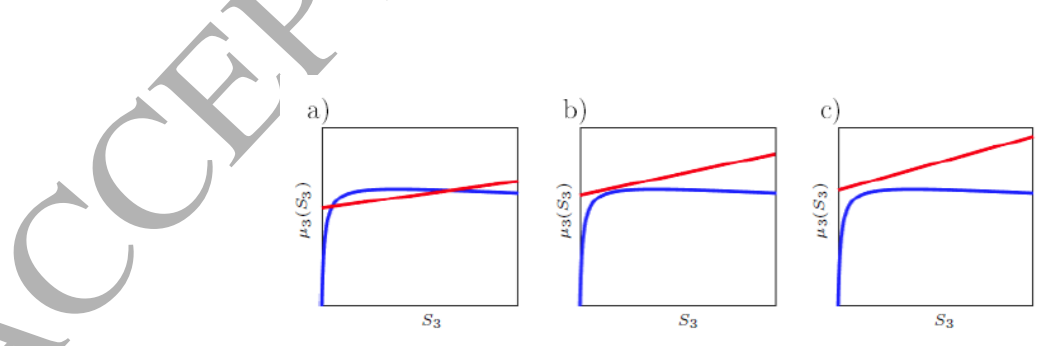

Figure 3: Possible cases of the intersection of functions $f\left(S_{3}\right)$ (blue lines), $g\left(S_{3}\right)$ (red lines), a) Two intersections, b) One intersection, c) No intersection. 
From equation (29), we can see that $S_{3}^{*}$ is accepted if, and only if, $S_{3}^{*}<S_{3 i n}^{*}$, because $X_{3}^{*}$ should be positive. Thus, the condition $f\left(S_{3 i n}^{*}\right)=g\left(S_{3 i n}^{*}\right)$ is crucial for evaluating if the solutions $S_{3}^{*}$ are accepted or not.

According to the sign of the equation $f\left(S_{3 i n}^{*}\right)-g\left(S_{3 i n}^{*}\right)$, we can investigate the values of $S_{3}^{*}$ with respect to those of $S_{3 i n}^{*}$. In the case of two intersection (Figure 3-a), we can deduce the sub-cases illustrated in Figure 4.
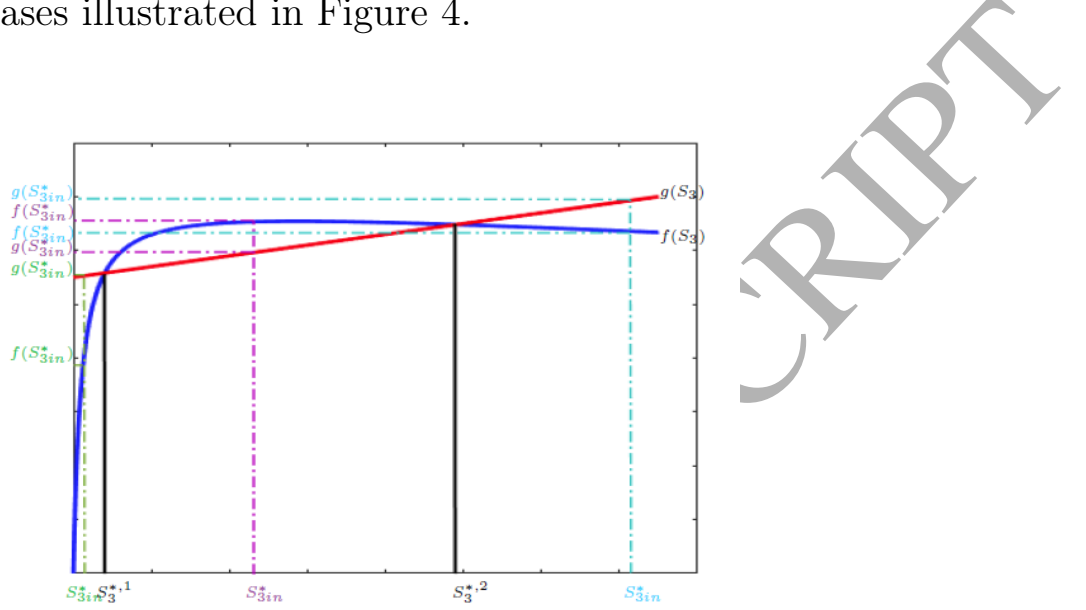

Figure 4: Possible positions of $S_{3 i n}^{*}$ in relation to $S_{3}^{* 1}$ and $S_{3}^{* 2}$.

When $f\left(S_{3 i n}^{*}\right)-g\left(S_{3 i n}^{*}\right)<0$, we have i) no solution if $S_{3 i n}^{*}<S_{3}^{*, 1}<S_{3}^{*, 2}$ (Figure 4 , green dashed lines); or ii) two solutions $S_{3}^{*, 1}>0$ and $S_{3}^{*, 2}>0$ if $S_{3}^{*, 1}<S_{3}^{*, 2}<S_{3 \text { in }}^{*}$ (Figure 4, cyan dashed lines).

If $f\left(S_{3 i n}^{*}\right)-g\left(S_{3 i n}^{*}\right)>0$, only one solution is accepted: $S_{3}^{*, 1}>0$ (Figure 4, magenta dashed lines). The solution $S_{3}^{*, 2}>S_{3 i n}^{*}$ gives $X_{3}^{*, 2}<0$ according to (29).

Substituting the feasible solutions $S_{3}^{*, i}$ in the equation (29), we deduce $X_{3}^{*, i}(i=1,2)$ :

$$
X_{3}^{*, i}=\frac{S_{3 i n}^{*}-S_{3}^{*, i}}{\alpha_{9}}
$$

Subsequently, $N^{*, i}(i \neq 1,2)$ are obtained from the equation (31) as:

$$
N^{*, i}=N_{i n}^{*}-\frac{\alpha_{10}}{\alpha_{9}}\left(S_{3 i n}^{*}-S_{3}^{*, i}\right)
$$

$N^{*, i}$ exist if and only if, $N_{i n}^{*}>\frac{\alpha_{10}}{\alpha_{9}}\left(S_{3 i n}^{*}-S_{3}^{*, i}\right)$.

Remark. We denote that:

$$
H\left(S_{3}\right)=f\left(S_{3}\right)-g\left(S_{3}\right)=\frac{M\left(S_{3}\right)}{K_{I, n h_{3}} \alpha_{9}\left(S_{3}^{2}+K_{I 3} S_{3}+K_{I 3} K_{S_{3}}\right)}
$$


$S_{3}^{*}$ can be, also, deduced from the numerator of $H\left(S_{3}\right)$, denoted $M\left(S_{3}\right)$, and defined by the following third order equation:

$$
M\left(S_{3}\right)=a_{3} S_{3}^{3}+a_{2} S_{3}^{2}+a_{1} S_{3}+a_{0}
$$

where

- $a_{3}=-D \lambda \alpha_{10}$

- $a_{2}=-D\left(K_{I, n h_{3}} \alpha_{9}+\lambda A \alpha_{9}+\lambda \alpha_{10} K_{I 3}\right)$

- $a_{1}=K_{I 3}\left(K_{I, n h_{3}} \alpha_{9}\left(\bar{\mu}_{3}-D\right)-D \lambda\left(A \alpha_{9}+\alpha_{10} K_{S_{3}}\right)\right)$

- $a_{0}=-D \alpha_{9} K_{S_{3}} K_{I 3}\left(\lambda A+K_{I, n h_{3}}\right)$

Using Cardan formulas, $M\left(S_{3}\right)$ is solved explicitly to obtain $S_{3}^{*}$. In this case, no more than two solutions are accepted in view of the constraint of positivity.

\section{Proposition 1.}

System (1)-(7) has at most 10 points of equilibrium:

$E_{1}=\left(\beta_{1} S_{i n}, \beta_{2} S_{i n}, 0,0,0,0, N_{i n}\right)$, washout equilibrium, which always exists.

$E_{2}=\left(\beta_{1} S_{i n}, S_{2}^{*}, S_{3}^{*}, 0, X_{2}^{*}, 0, N^{*}\right)$, which exists if and only if, $D<\bar{\mu}_{2}$.

$E_{3}^{1}=\left(\beta_{1} S_{\text {in }}, S_{2}^{*}, S_{3}^{*, 1}, 0, X_{2}^{*}, X_{3}^{*, 1}, N^{*, 1}\right)$, which exists if and only if, $D<\bar{\mu}_{2}$ and $X_{3}^{*, 1}>0$ and $\alpha_{6} X_{2}^{*}>\alpha_{9} X_{3}^{*, 1}$ and $N_{i n}+\alpha_{7} X_{2}^{*}>\alpha_{10} X_{3}^{*, 1}$.

$E_{3}^{2}=\left(\beta_{1} S_{i n}, S_{2}^{*}, S_{3}^{*, 2}, 0, X_{2}^{*}, X_{3}^{*, 2}, N^{*, 2}\right)$, which exists if and only if, $D<\overline{\mu_{2}}$ and $X_{3}^{*, 2}>0$ and $\alpha_{6} X_{2}^{*}>\alpha_{9} X_{3}^{*, 2}$ and $N_{\text {in }}+\alpha_{7} X_{2}^{*}>\alpha_{10} X_{3}^{*, 2}$.

$E_{4}=\left(S_{1}^{*}, \beta_{2} S_{i n}, S_{3}^{*}, X_{1}^{*}, 0,0, N^{*}\right)$, which exists if and only if, $D<\bar{\mu}_{1}$ and $N_{\text {in }}-$ $\alpha_{2} X_{1}^{*}>0$.

$E_{5}^{1}=\left(S_{1}^{*}, \beta_{2} S_{i n}, S_{3}^{* 1}, X_{1}^{*}, 0, X_{3}^{*, 1}, N^{*, 1}\right)$, which exists if and only if, $D<\bar{\mu}_{1}$ and $X_{3}^{*, 1}>0$ and $\alpha_{3} X_{1}^{*}>\alpha_{9} X_{3}^{*, 1}$ and $N_{i n}-\alpha_{2} X_{1}^{*}>\alpha_{10} X_{3}^{*, 1}$.

$E_{5}^{2}=\left(S_{1}^{*}, \beta_{2} S_{i n}, S_{3}^{*, 2}, X_{1}^{*}, 0, X_{3}^{*, 2}, N^{*, 2}\right)$, which exists if and only if, $D<\bar{\mu}_{1}$ and $X_{3}^{*, 2}>0$ and $\alpha_{3} X_{1}^{*}>\alpha_{9} X_{3}^{*, 2}$ and $N_{i n}-\alpha_{2} X_{1}^{*}>\alpha_{10} X_{3}^{*, 2}$.

$E_{6}=\left(S_{1}^{*}, S_{2}^{*}, S_{3}^{*}, X_{1}^{*}, X_{2}^{*}, 0, N^{*}\right)$, which exists if and only if, $D<\bar{\mu}_{1}$ and $D<\bar{\mu}_{2}$ and $N_{\text {in }}-\alpha_{2} X_{1}^{*}+\alpha_{7} X_{2}^{*}>0$.

$E_{7}^{1}=\left(S_{1}^{*}, S_{2}^{*}, S_{3}^{*, 1}, X_{1}^{*}, X_{2}^{*}, X_{3}^{*, 1}, N^{*, 1}\right)$, which exists if and only if, $D<\bar{\mu}_{1}$ and $D<\bar{\mu}_{2}$ and $X_{3}^{*, 1}>0$ and $\alpha_{3} X_{1}^{*}+\alpha_{6} X_{2}^{*}>\alpha_{9} X_{3}^{*, 1}$ and $N_{\text {in }}-\alpha_{2} X_{1}^{*}+\alpha_{7} X_{2}^{*}>\alpha_{10} X_{3}^{*, 1}$.

$E_{7}^{2}=\left(S_{1}^{*}, S_{2}^{*}, S_{3}^{*, 2}, X_{1}^{*}, X_{2}^{*}, X_{3}^{*, 2}, N^{*, 2}\right)$, which exists if and only if, $D<\bar{\mu}_{1}$ and $D<\bar{\mu}_{2}$ and $X_{3}^{*, 2}>0$ and $\alpha_{3} X_{1}^{*}+\alpha_{6} X_{2}^{*}>\alpha_{9} X_{3}^{*, 2}$ and $N_{i n}-\alpha_{2} X_{1}^{*}+\alpha_{7} X_{2}^{*}>\alpha_{10} X_{3}^{*, 2}$. 
The diagram of Figure 5 summarizes the different cases corresponding to the system equilibria.
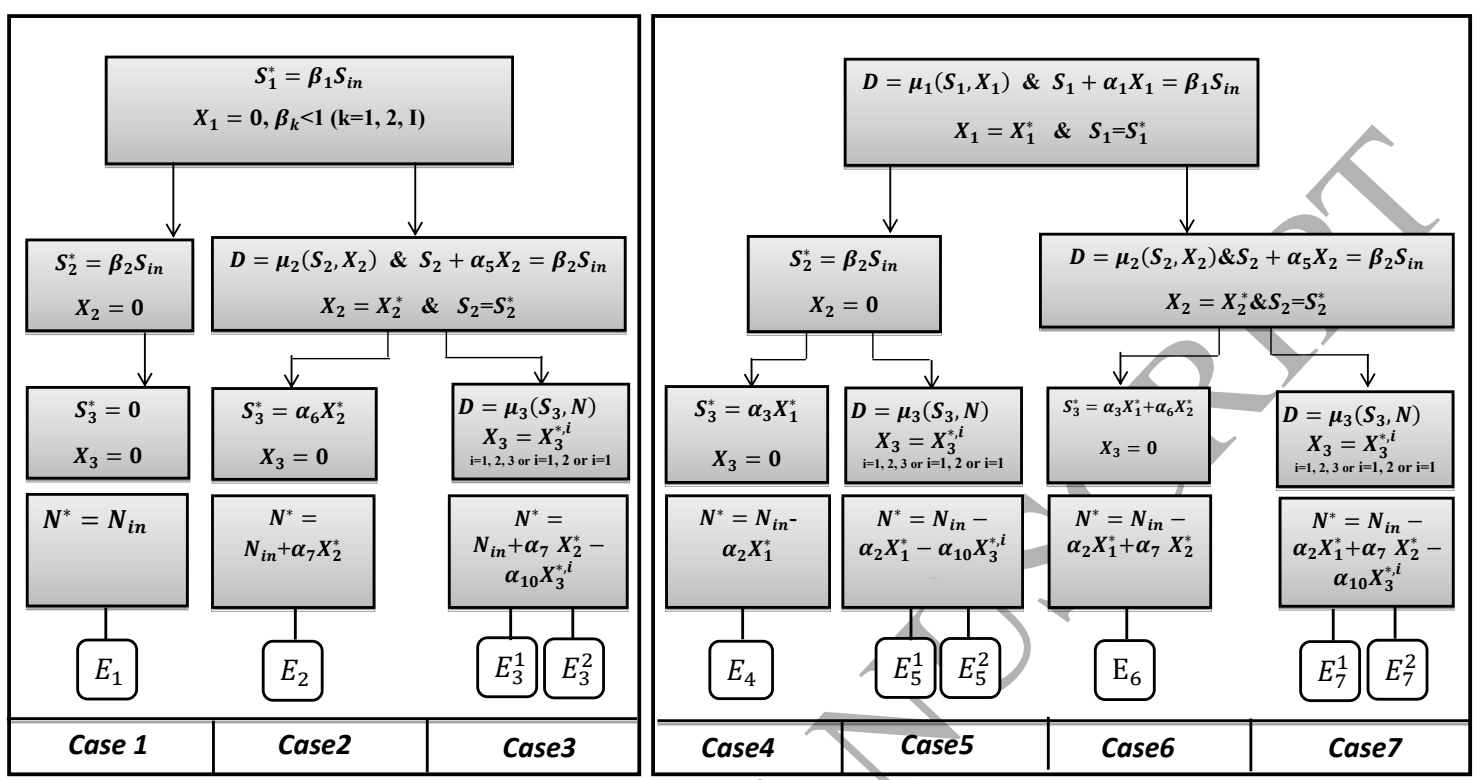

Figure 5: Diagram summarizing the equilibria of system (1)-(7). The system has at most 10 points of equilibrium.

Proof. See proof in Appendix A.

\subsection{Numerical investigation of the stability}

The equilibria of the MAD model are dependent on the values of the operating parameters $D, S_{i n}, N_{\text {in }}$ (Figure 5). Obviously, they greatly affect equilibrium stability and thus the process operation.

To establish whether the equilibria of the MAD model are stable or not, the Jacobian matrix is computed for each equilibrium. If we write the system in the variables $\left[S_{1}, X_{1}, S_{2}, X_{2}, S_{3}, X_{3}, N\right]$, the Jacobian matrix will have block-diagonal structure:

$$
J=\left(\begin{array}{ccc}
A & 0_{2 * 2} & 0_{2 * 3} \\
0_{2 * 2} & B & 0_{2 * 3} \\
E & F & C
\end{array}\right)
$$

In the case where only one or two bacteria at most coexist, the nature of the equilibria can be investigated analytically. The eigenvalues of the Jacobian matrix are those of the matrices A, B and C. We use the Routh-Hurwitz criterion to study the stability 
of the matrix $\mathrm{C}$, which is of third-order (see Appendix B).

When all bacterial consortiums coexist, the analytical study of equilibria and their stability becomes very complicated: it is necessary to use numerical tools to find the nature of equilibria. Hereafter, we investigate numerically the equilibria stability with respect to the changes of operating parameters values. Considering a large range of operating parameter values, we construct the operating diagram (see Appendix C) of the MAD model $[18,19]$.

We fixed the inorganic nitrogen inlet at the average value taken in the experiments $N_{i n}=0.011 M$ [16]. Then, for several values of $D$ and $S_{i n}$ we computed equilibria and the corresponding Jacobian matrix. The nature: stability and existence were assigned to each of the 10 equilibria. This enabled us to establish a set of existence-stability combinations for each pair $\left(D, S_{i n}\right)$ as summarized in Table 3. Each combination is plotted as a colored region $\mathcal{J}_{n}$ (where $n$ is the number of the combinations) in the operating diagram.

All concentrations are in $\left(g C O D . L^{-1}\right)$, except the inorganic carbon and the nitrogen which are in $(M)$. The bacterial growth and dilution rates are in $\left(\right.$ day $\left.^{-1}\right)$. Nominal values of the model parameters are reported in Table 2. 
Table 2: Nominal parameter values used by Mairet et al. [16].

\begin{tabular}{|c|c|c|c|}
\hline & Parameter & & Unit \\
\hline$\beta_{1}=0.3$ & $\beta_{2}=0.4$ & $\alpha_{1}=12.5$ & $g C O D \cdot g C O D^{-1}$ \\
\hline$\alpha_{3}=11.5$ & $\alpha_{5}=9.1$ & $\alpha_{6}=8.1 \quad \alpha_{9}=20$ & \\
\hline$K_{S_{1}}=2.11$ & $K_{S_{2}}=0.056$ & & \\
\hline$\alpha_{2}=0.0062$ & $\alpha_{4}=0.03$ & $\alpha_{7}=0.054 \quad \alpha_{8}=0.03$ & \\
\hline$\alpha_{10}=0.0062$ & $\alpha_{11}=0.30$ & $\alpha_{12}=0.20$ & \\
\hline $\bar{\mu}_{1}=0.30$ & $\bar{\mu}_{2}=0.053$ & $\bar{\mu}_{3}=0.14 \quad K_{L} a$ & \\
\hline$K_{S_{3}}=0.02$ & $K_{I 3}=16.4$ & & $g C O D \cdot L^{-1}$ \\
\hline$K_{C}=4.9 e-7$ & $K_{N}=1.1 e-9$ & & $M$ \\
\hline$V_{l i q}=1$ & $V_{\text {gas }}=0.1$ & & $L$ \\
\hline$K_{H, C O_{2}}=2.7 e-2$ & & & M.bar ${ }^{-1}$ \\
\hline$k_{p}=5 e 4$ & & & $L . d^{-1} \cdot b a r^{-1}$ \\
\hline$R=8.31 e-2$ & & & $\operatorname{bar} \cdot M^{-1} \cdot K^{-1}$ \\
\hline$T_{o p}=308.15$ & & & $K$ \\
\hline
\end{tabular}

One of the main interests of the operating diagram is to highlight, which equilibria are stable for a given combination of inputs parameters $\left(D, S_{i n}\right)$.

In order to limit the number of regions plotted in a diagram, we have grouped together the very similar regions, which are just different by the existence of an unstable point. At a neutral $\mathrm{pH}$, it is for instance the case in $\mathcal{J}_{4}$ where $E_{3}^{1}$ and $E_{5}^{1}$ are unstable if they exist ( $\mathrm{X}$ in Table 6 ). 


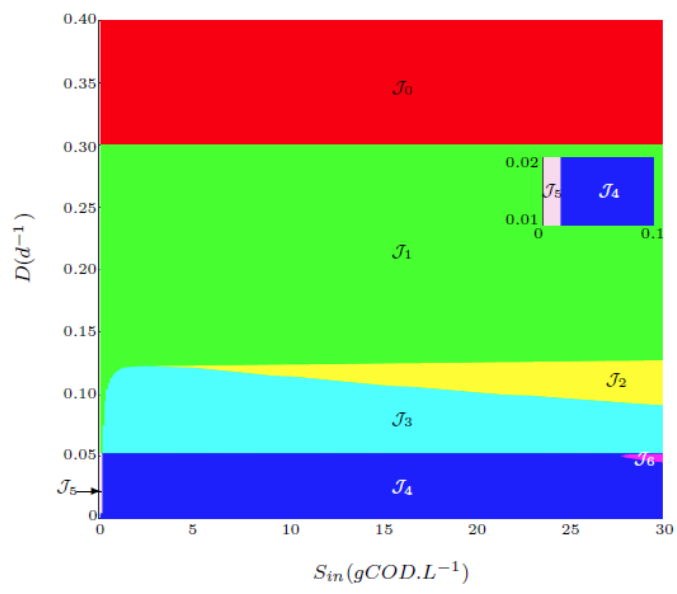

Figure 6: Operating diagram showing the behavior of equilibria as a function of $D$ and $S_{\text {in }}$ at $p H=7, N_{i n}=0.011 M$. The area $\mathcal{J}_{5}$ is too small because it exists for only small values of $S_{i n}$.

Table 3: Existence and nature of equilibria according to $S_{i n}$ and $D$ at $p H=7$ : Unstable (U), Stable $(\mathrm{S})$, unstable or does not exist (X), Equilibrium does not exist (no sign).

\begin{tabular}{|c|c|c|c|c|c|c|c|c|c|c|}
\hline Area & $E_{1}$ & $E_{2}$ & $E_{3}^{1}$ & $E_{3}^{2}$ & $E_{4}$ & $E_{5}^{1}$ & $E_{5}^{2}$ & $E_{6}$ & $E_{7}^{1}$ & $E_{7}^{2}$ \\
\hline $\mathcal{J}_{0}$ & $S$ & & & & & & & & & \\
\hline $\mathcal{J}_{1}$ & $U$ & & & & & & & & & \\
\hline $\mathcal{J}_{2}$ & $U$ & & & & & $S$ & $U$ & & & \\
\hline $\mathcal{J}_{3}$ & $U$ & & & & $U$ & $S$ & & & & \\
\hline $\mathcal{J}_{4}$ & $U$ & & & & $U$ & $X$ & & $U$ & $S$ & \\
\hline $\mathcal{J}_{5}$ & & & & & $U$ & & & $S$ & & \\
\hline $\mathcal{J}_{6}$ & $U$ & & $U$ & & $U$ & $U$ & & $S$ & $S$ & $U$ \\
\hline
\end{tabular}

Figure 6 shows seven areas; hence, seven possible combinations of the equilibria can be obtained when $\mathrm{pH}$ has a neutral value (see Table 3 ).

The transition from one region to another, resulting from a change in the model inputs $S_{\text {in }}$ and// or $D$ exhibits what is called equilibria bifurcation (appearance, disappearance or coalescence of equilibria). For example, when reading the Figure 6 from the bottom to the top, we notice that an increase of $\mathrm{D}$ for a fixed value of $S_{\text {in }}=20 \mathrm{gCOD} \cdot \mathrm{L}^{-1}$ leads to the appearance of several regions: $\mathcal{J}_{4}, \mathcal{J}_{3}$ and $\mathcal{J}_{2}$, respectively, evidencing some changes in the nature of the equilibria (as reported in Table 3$)$. When $D$ is very low $(D<0.04)$, we obtain the area $\left(\mathcal{J}_{4}\right)$ where only the 
functioning equilibrium $E_{7}^{1}$ is stable. A slight increase of $D(0.04<D<0.05)$ leads to area $\left(\mathcal{J}_{6}\right)$ : the equilibrium $E_{6}$ changes its nature and becomes stable.

An area $\left(\mathcal{J}_{5}\right)$ appears when $S_{\text {in }}$ is too low and $D$ takes small values $(0.01<D<$ 0.05 )(see the zoom, right Figure 6). In this area the equilibrium $E_{6}$ is stable: the consortium $X_{3}$ is washed out due to the nitrogen deficiency. If, however, $D$ belongs to the range $(0.05<D<0.07)$, the system operates in the area $\left(\mathcal{J}_{3}\right): X_{2}$ is washed out and $E_{5}^{1}$ is stable. An increasing of $\mathrm{D}$ with $0.02 d^{-1}$ leads to area $\mathcal{J}_{2}$ : $E_{5}^{1}$ is still stable, $E_{4}$ changes its nature and becomes stable and the stable equilibrium $E_{5}^{2}$ appears. This is due to the bifurcation of the equilibria.

Five of the seven combinations involved one stable equilibrium: $E_{7}^{1}\left(\mathcal{J}_{4}\right)$ or $E_{6}$ $\left(\mathcal{J}_{5}\right)$ when the three reactions occur simultaneously $\left(D<\min \left(\bar{\mu}_{1}, \bar{\mu}_{2}, \bar{\mu}_{3}\right)\right), E_{5}^{1}\left(\mathcal{J}_{3}\right)$ when $D$ exceeds $\bar{\mu}_{2}$ (only the reactions (R1) and (R2) take place) and $E_{4}\left(\mathcal{J}_{1}\right)$ when $D$ is higher than $\bar{\mu}_{3}$ (only the reaction (R1) take place). When no reaction occurs $\left(D>\max \left(\bar{\mu}_{1}, \bar{\mu}_{2}, \bar{\mu}_{3}\right)\right)$, the washout equilibrium $E_{1}\left(\mathcal{J}_{0}\right)$ becomes stable.

Areas $\mathcal{J}_{2}$ and $\mathcal{J}_{6}$ present the bi-stability of the system, where it could tend towards the interior equilibrium or to the washout one, depending on the initial concentration of the methanogens $\left(X_{3_{0}}\right)$. Such operating areas appear when the dilution rate $(D)$ and/or the organic load $\left(S_{\text {in }}\right)$ reach(es) high levels.

The operating diagram is, then, a good indicator of the risk of AD process failure. It provides good informations about the qualitative properties of the model and the optimum range of operating conditions.

The region $\mathcal{J}_{4}$ can be defined as a normal operation area, where all the bacteria coexist. It is then the ideal zone to ensure the digester performances.

Areas $\mathcal{J}_{5}$ and $\mathcal{J}_{3}$ correspond to the system operation with the washout of $X_{3}$ and $X_{2}$, respectively. The area $\mathcal{J}_{1}$ predicts the washout of both $X_{2}$ and $X_{3}$, and only the consortium $X_{1}$ exists. All the bacteria are washed out in the area $\mathcal{J}_{0}$ that exhibits a high risk of process destabilization .

When the digester operates in the bi-stability areas $\left(\mathcal{J}_{2}, \mathcal{J}_{6}\right)$, implementation of a closed-loop regulation system is needed to avoid the washout equilibrium.

\subsection{Bifurcation phenomena}

In order to highlight the origin of bi-stability, we attempt to show the behaviour of $S_{3}, X_{3}$ and $N$ when Figure 6 is browsed either vertically for a constant organic load or horizontally for a constant dilution rate.

Figure 7 shows the behavior of the existing equilibrium components, capable of changing their nature in accordance with change in the dilution rate. 
a)

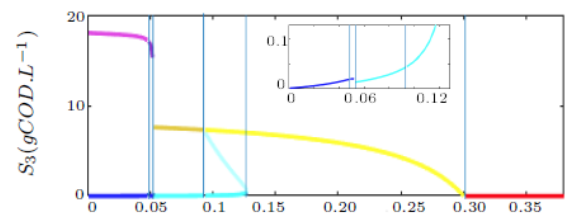

b)

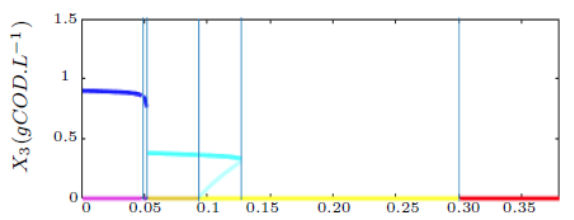

c)

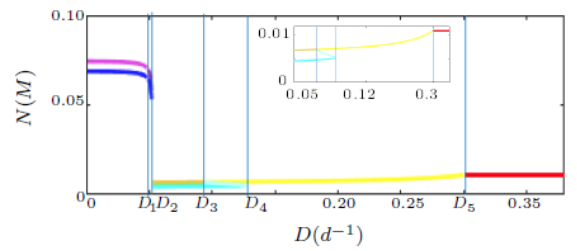

Figure 7: The components of the equilibrium points with respect to the $D$ at $p H=7, S_{\text {in }}=$ $28.5 \mathrm{gCOD} . \mathrm{L}^{-1}$ and $N_{\text {in }}=0.011 \mathrm{M}$.
$E_{6}$ unstable
$E_{7}^{1}$ stable $E_{6}$ stable
- $E_{4}$ unstable
- $E_{5}^{1}$ stable $E_{4}$ stable $E_{1}$ stable
$E_{5}^{2}$ unstable

The critical values of $D$ are:

$$
D_{1}=0.040, D_{2}=\bar{\mu}_{2}, D_{3}=0.09, D_{4} \approx \bar{\mu}_{3}, D_{5}=\bar{\mu}_{1}
$$

with

- $S_{3 i n, t o t a l}^{*}\left(D_{1}\right) \rightleftharpoons S_{3}^{i, *}\left(D_{1}\right)$, with $S_{3}^{i, *}=\alpha_{3} X_{1}^{*}+\alpha_{6} X_{2}^{*}-\alpha_{9} X_{3}^{i, *}(i=1,2)$ thus $X_{3}^{i, *}\left(D_{1}\right)=0($ case 7, Figure 5$)$,

- $D_{2}=\mu_{2}\left(\beta_{2} S_{i n}, 0\right)$,

- $S_{3 \text { in }, 1}^{*}\left(D_{3}\right)=S_{3}^{i, *}\left(D_{3}\right)$, with $S_{3}^{i, *}=\alpha_{3} X_{1}^{*}-\alpha_{9} X_{3}^{i, *}(i=1,2)$, thus $X_{3}^{i, *}\left(D_{3}\right)=0$ (case 5, Figure 5),

- $D_{4}=\mu_{3}(0,0)$, 


$$
\text { - } D_{5}=\mu_{1}\left(\beta_{1} S_{i n}, 0\right) \text {. }
$$

As can be seen in figure 7 , a small change in the dilution rate results in a change of the nature of some model equilibria. For a fixed value of the organic load $\left(S_{i n}\right)$ increasing $D$ may decrease the concentration of $X_{3}$ of the stable equilibrium (Figure 7-b).

It leads to the accumulation of the substrates $S_{1}$ and $S_{2}$, and then to a considerable production of VFAs $\left(S_{3}\right)$ from reaction 1 (R1) and/or reaction 2 (R2) (see the zoom, top Figure 7-a). This boosts the amount of ionized and non-ionized forms of the VFAs in the reaction medium. Both forms are present with balanced concentrations because the $\mathrm{pH}$ is assumed to be neutral. In such case, a potential inhibition of methanogenic bacteria $\left(X_{3}\right)$ occurs by an accumulation of VEAs. Hence, the reaction 3 (R3) will be affected by VFA accumulation: increase of $S_{3}$ supported by a significant decrease of $X_{3}$.

This risk of inhibition becomes more severe as the organic load $\left(S_{i n}\right)$ increases. The latter induces high release of ammonia, which explains the appearance of the areas $\left(\mathcal{J}_{2}\right)$ and $\left(\mathcal{J}_{6}\right)$ where, respectively, the equilibria $E_{4}, E_{5}^{1}$ and $E_{6}, E_{7}^{1}$ are stable.

It is clear from Figure 7 -c that the nitrogen concentration of the stable equilibria $\left(N^{*}\right)$ is dependent on the value of $D$. The value of nitrogen at steady-states $N^{*}$ is higher when $D$ is lower than the maximum growth rate of $X_{2}\left(\bar{\mu}_{2}\right)$. This is due to the achievement of the reaction (R2) that releases the nitrogen from the breakdown of proteins. This phenomena cannot be observed beyond $\bar{\mu}_{2}$ because, in this case, the reactor contains only $N_{i n}$ as a nitrogen source.

Thus, when $D<\bar{\mu}_{2}$, the concentration of $N^{*}$ decreases to satisfy high bacterial needs. However, when $D>\bar{\mu}_{2}$, bacterial needs decreases because the Hydraulic Retention Time (HRT) is small. Therefore, nitrogen consumption is not high and $N^{*}$ concentration increases (see the zoom, top Figure 7-c).

This reveals that increasing $D$ may increase the risk of process failure by providing an excess of $S_{3}$, not an excess of nitrogen (because $\mathrm{D}$ alleviates the ammonia stress [11]).

Figures 8 and 9 present the behavior of the components of existing equilibria, capable to change their nature when the organic load $\left(S_{i n}\right)$ varies, and D is lower and higher than $\bar{\mu}_{2}$, respegtively.

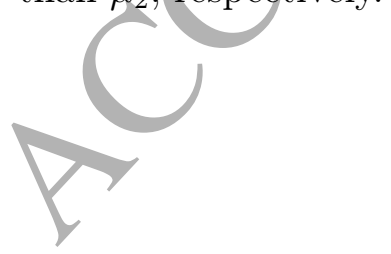



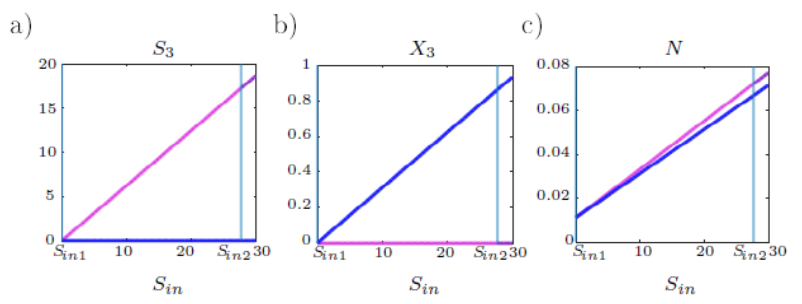

Figure 8: The components of the equilibrium points with respect to $S_{\text {in }}$ at $p H=7, D=0.0301 d^{-1}$ and $N_{\text {in }}=0.011 M$.

The critical values of the organic load $S_{i n 1}$ and $S_{i n 2}$ are around $0.02 g C O D . L^{-1}$ and $28.5 g C O D . L^{-1}$, respectively, with:

$$
\begin{aligned}
& S_{3 i n, t o t a l}^{*}\left(S_{i n 1}\right)>S_{3}^{i, *}\left(S_{i n 1}\right), \text { thus } X_{3}^{i, *}\left(S_{i n 1}\right)>\theta(\text { case } 7, \text { Figure } 5) . \\
& \left.S_{3 i n, t o t a l}^{*}\left(S_{i n 2}\right)=S_{3}^{i, *}\left(S_{i n 2}\right), \text { thus } X_{3}^{i, *}\left(S_{i n 2}\right)=0 \quad \text { (case } 7, \text { Figure } 5\right) .
\end{aligned}
$$

For a fixed value of $D<\bar{\mu}_{2}$, the increase of the organic load yields high concentrations of the substrates $S_{1}$ and $S_{2}$ in the medium. The concentrations of the bacteria and inorganic nitrogen $N^{*}$ of the stable equilibria $\left(E_{6}\right.$ before $S_{i n 1}, E_{7}^{1}$ before $S_{i n 2}$ and $E_{6} / E_{7}^{1}$ after exceeding $S_{i n 2}$ ) increase accordingly. This can be regarded as abnormal behavior because no inhibition is seen even though the ammonia concentration is high and VFAs have accumulated.
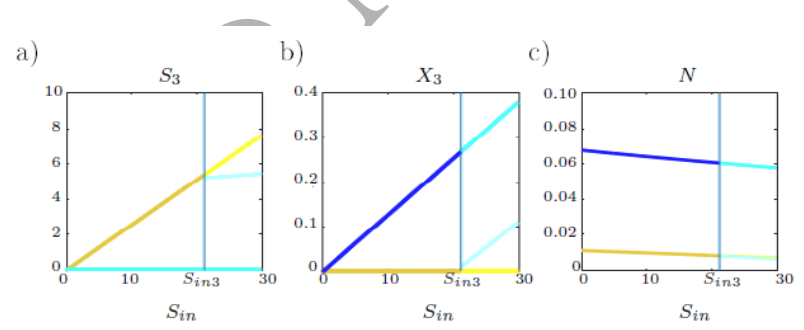

Figure 9: The components of the equilibrium points with respect to $S_{i n}$ at $p H=7, D=0.101 d^{-1}$ and $N_{i n}=0.011 M$.

If $D$ is fixed at a constant value beyond $\bar{\mu}_{2}\left(X_{2}\right.$ washed out $)$, the critical value of the organic load is about $S_{i n 3}=21.5 \mathrm{gCOD} . \mathrm{L}^{-1}$, with:

$$
S_{3 i n, 1}^{*}\left(S_{i n 3}\right)=S_{3}^{i, *}\left(S_{i n 3}\right) \text {, thus } X_{3}^{i, *}\left(S_{i n 3}\right)=0 \quad(\text { case 5, Figure 5). }
$$


The increase of $S_{i n}$ leads to an increase of $S_{3}$ and a decrease of $N$ of the stable equilibria ( $E_{5}^{1}$ before $S_{i n_{3}}$ and $E_{4} / E_{5}^{1}$ after exceeding $S_{i n_{3}}$ ). Consequently, $X_{3}$ increases by consuming $S_{3}$ and the nitrogen coming from $N_{i n}$.

These results show that a small change to the control parameters may lead to a noteworthy change in the MAD behavior. Special attention must be given to their values to avoid process failure.

\section{Global behavior of the system}

\subsection{Influence of $p H$}

It is well known that $\mathrm{pH}$ plays a key role in Anaerobic Digestion. It can, thus, cause the imbalance of ions at certain values.

$\mathrm{pH}$ may suddenly rise during the treatment of protein materials rich in nitrogen, particularly at low buffer capacity. At high levels, $\mathrm{pH}$ promotes the release of unionized ammonia toxic to methanogens, while at low levels, free acids formation is significant and bacteria are inhibited by VFAs accumulation.

Simulation and experimental results presented in Mairet el al. [16] stress that the $\mathrm{pH}$ can vary between 6.5 and 7.5 throughout the digestion of chlorella vulgaris microalgae.

To emphasize $\mathrm{pH}$ influence on the $\mathrm{AD}$ of such protein-rich substrates, we establish below the operating diagram of the MAD model in an acidic medium $(\mathrm{pH}=6.5)$ as well as in a basic medium ( $\mathrm{pH}=7.5)$. In thefollowing simulations, the representation of the operating diagram is made with $D$ ranging from 0 to 0.15 . 
Table 4: Existence of equilibria and stability at $p H=6.5, N_{i n}=0.011 M$ : unstable (U), stable (S), unstable or does not exist (X), equilibrium does not exist (no sign).

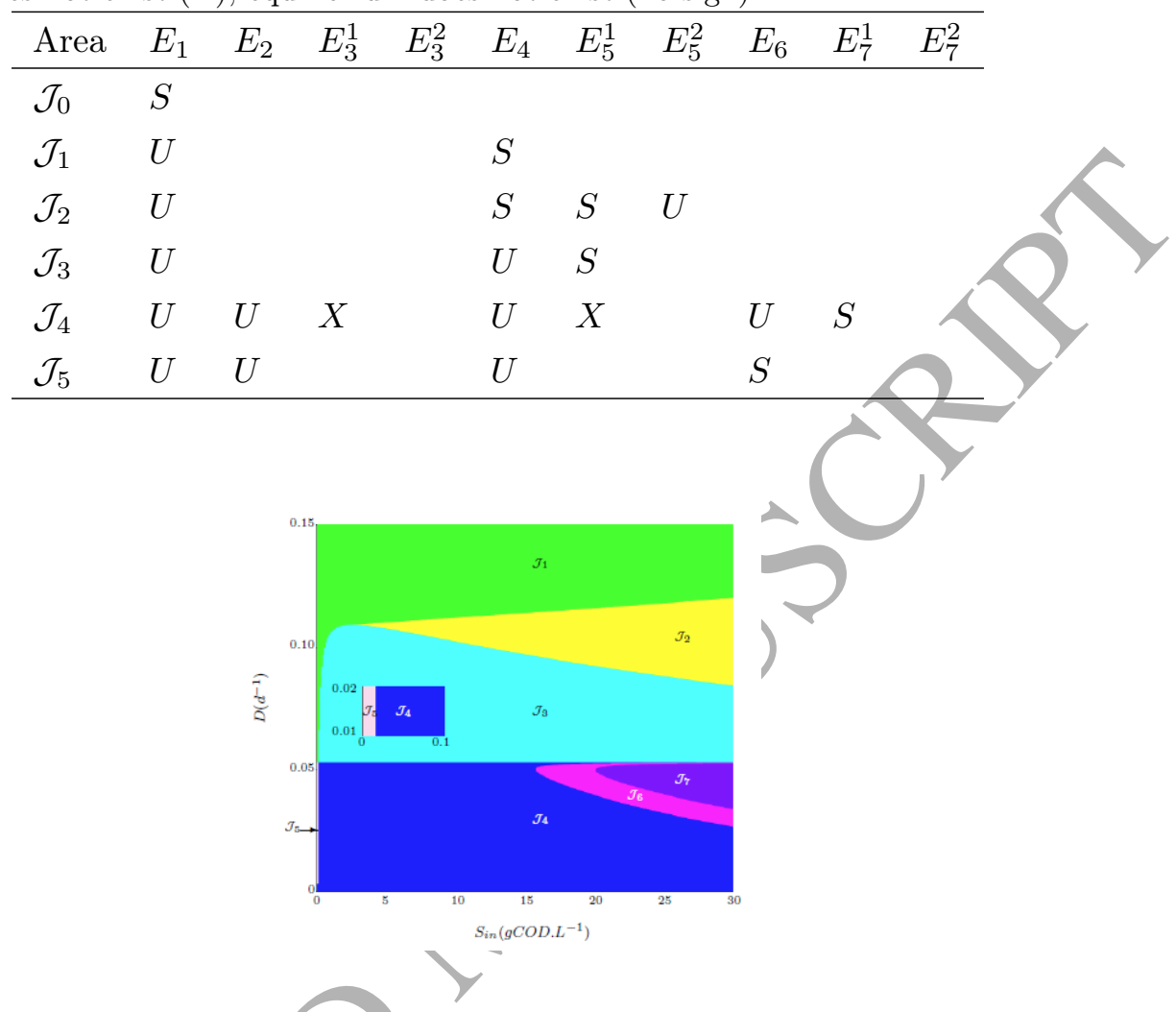

Figure 11: Operating diagram - behavior of equilibria as a function of varying $D$ and $S_{\text {in }}$ at $p H=7.5, N_{i n}=0.011 M$. The area $\mathcal{J}_{5}$ is too small in the OD because it exists for only small values of $S_{i n}$.

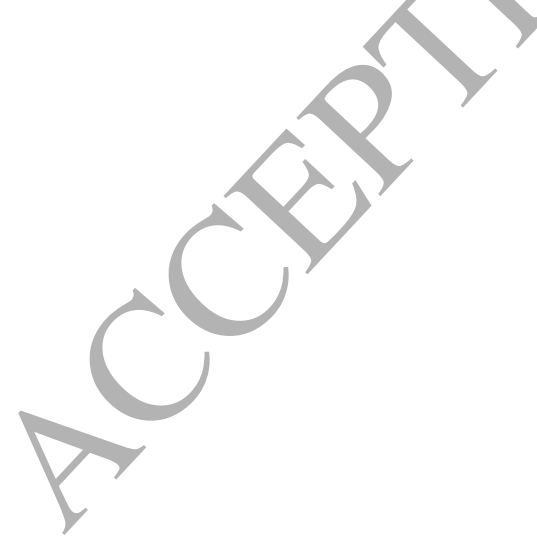


Table 5: Existence of equilibria and stability at $p H=7.5$ : unstable (U), stable $(\mathrm{S})$, unstable or does not exist $(\mathrm{X})$, equilibrium does not exist (no sign).

\begin{tabular}{ccccccccccc}
\hline Area & $E_{1}$ & $E_{2}$ & $E_{3}^{1}$ & $E_{3}^{2}$ & $E_{4}$ & $E_{5}^{1}$ & $E_{5}^{2}$ & $E_{6}$ & $E_{7}^{1}$ & $E_{7}^{2}$ \\
\hline $\mathcal{J}_{0}$ & $S$ & & & & & & & & & \\
$\mathcal{J}_{1}$ & $U$ & & & & $S$ & & & & & \\
$\mathcal{J}_{2}$ & $U$ & & & & $S$ & $S$ & $U$ & & & \\
$\mathcal{J}_{3}$ & $U$ & & & & $U$ & $S$ & & & & \\
$\mathcal{J}_{4}$ & $U$ & $U$ & $X$ & & $U$ & $X$ & & $U$ & $S$ & \\
$\mathcal{J}_{5}$ & $U$ & $U$ & & & $U$ & & & $S$ & & \\
$\mathcal{J}_{6}$ & $U$ & $U$ & $U$ & & $U$ & $U$ & & $S$ & $S$ & $U$ \\
$\mathcal{J}_{7}$ & $U$ & $U$ & $U$ & $U$ & $U$ & $U$ & & $S$ & $S$ & $U$ \\
\hline
\end{tabular}

By comparing the operating diagrams representing an acidic $\mathrm{pH}$ (Figure 10) and a neutral pH (Figure 6), we find the same areas except for $\mathcal{J}_{6}$, a bi-stability area undesirable from a practical point of view. Moreover, when the $\mathrm{pH}$ takes a neutral value, we can see that the bi-stability area $\mathcal{J}_{2}$ appears at a dilution value that causes a total washout of $X_{3}$.

The risk of process failure at $p H=7$ seems to be higher than that observed at $p H=6.5$. Such a result does not agree with the numerous studies in the literature that prove the sensitivity of methanogens to high VFAs concentrations [20, 21]. Henceforth, the value chosen for the inhibition constant $K_{I 3}$ associated to VFA $\left(S_{3}\right)$ and proposed in Mairet et al. prevents the occurrence of an inhibition by an excess of VFAs. We have plotted in Figure 12 the pattern of methanogens growth rate for a small value of $K_{I 3}$. 


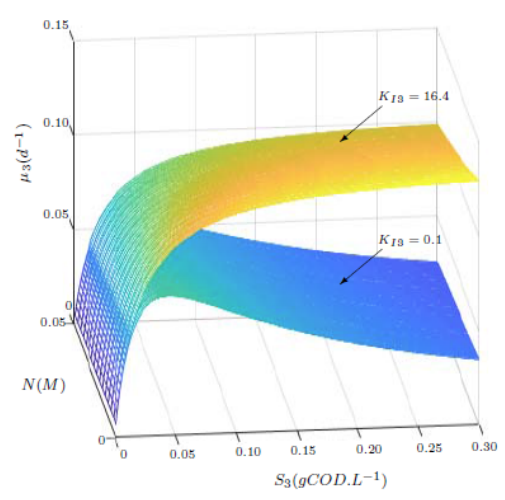

Figure 12: Methanogen growth rates

From Figure 12, it is clear that the $X_{3}$ growth rate with the $K_{I 3}$ value reported in Mairet et al. [16] exhibits a Monod-like behavior. The inhibition of growth is only observed if smaller value of $K_{I 3}$ are used (Haldane function). This confirms our assumption and points out a significant limitation of the MAD model.

The comparison of Figure 11 with Figure 6 highlights new area $\mathcal{J}_{7}$ in a basic reaction medium, Figure 11 shows that the areas $\mathcal{J}_{4}$ and $\mathcal{J}_{3}$ narrow and the bistability zones are wider.

Indeed, when $D<\bar{\mu}_{2}$, the increase of $S_{\text {in }}$ leads to bi-stability $\left(\mathcal{J}_{6}\right.$ to $\left.\mathcal{J}_{7}\right)$. This is due to the continuous increase in the amount of nitrogen produced from (R2) and promoted by the $\mathrm{pH}$. When D exceeds $\bar{\mu}_{2}$, this risk is halved because (R2) is no longer active. In summary, the higher the organic load, the greater amount of nitrogen in the medium. In particular, at a $\mathrm{pH}$ of 7.5 , there is a shift leading to a higher Free Ammonia production, increasing the risk of process failure.

\subsection{Biogas yield}

The biogas flow rate is defined in Mairet et al. [16] by the equation (15). At equilibrium, biogas flow rate can be written as:

$$
q_{g a s}^{*}=k_{p}\left(P_{C H_{4}}^{*}+P_{C O_{2}}^{*}-P_{a t m}\right)
$$

$P_{\mathrm{CO}_{2}}^{*}$ and $P_{\mathrm{CH}}^{*}$ can be deduced by setting the right-hand sides of equations (8), (9) and (10) equal to zero. From the first equation, we can define $C^{*}$ depending on the model inputs and outputs:

$$
C^{* i}=C_{i n}+\alpha_{4} X_{1}^{*}+\alpha_{8} X_{2}^{*}+\alpha_{12} X_{3}^{* i}-\frac{\rho_{C O 2}}{D}
$$


By replacing (13), (14) and (33) in the equations (9) and (10), we get the following subsystem of two equations:

$$
\left\{\begin{array}{l}
-k_{p} P_{C O_{2}}^{2}-P_{C O_{2}}\left(k_{p} P_{C H_{4}}-k_{p} P_{a t m}+k_{L} a K_{H, C O_{2}} V_{l i q} R T_{o p}\right)+\frac{h}{K_{C}+h} k_{L} a V_{l i q} \cdots \\
\ldots R T_{o p} C^{*}\left(X_{1}^{*}, X_{2}^{*}, X_{3}^{*}, P_{C O_{2}}, P_{C H_{4}}\right)=0 \\
-k_{p} P_{C H_{4}}^{2}-k_{p} P_{C H_{4}}\left(P_{C O_{2}}-P_{a t m}\right)+\alpha_{11} \mu_{3} X_{3}^{* i} V_{l i q} R T_{o p}=0
\end{array}\right.
$$

Solutions of (35) allows the calculation of the biogas flow rate at equilibrium (Figure 13).

Figure 13: Biogas flow rate as a function of $\left(D, S_{i n}\right)$ changes at $p H=7, N_{i n}=0.011 M$.

Biogas yield shown in Figure 13 is highly dependent on the values of control parameters. For a fixed yalue of nitrogen inlet concentration $N_{i n}$, optimum biogas production is obtained for a dilution rate lower than the maximum growth rate $\bar{\mu}_{2}$. Moreover, a significant enhancement of biogas yield takes place when $S_{\text {in }}$ is high (yellow zone). It matches the area in which all bacteria coexist $\left(\mathcal{J}_{4}\right.$ and $\left.\mathcal{J}_{6}\right)$. The maximum production is, then, obtained when the equilibria $E_{7}^{1}$ and $E_{6}$ are stable.

However, when $D$ is greater than $\bar{\mu}_{2}\left(\mathcal{J}_{3}\right)$ less production of biogas is observed. Similarly, the highest yield is reached in the bi-stability area $\left(E_{5}^{1}\right.$ and $\left.E_{4}\right)$ when we operate around the interior equilibrium.

Otherwise, no biogas production is observed beyond the area $\mathcal{J}_{3}$ due to the washout of methanogen bacteria.

Hence, we must focus on a suitable choice of $X_{3_{0}}$ in the area of bi-stability in order to avoid the inhibition of bacteria on the one hand and, to ensure the maximum production of biogas on the other. 


\subsection{Relationship between $S_{i n}, D$ and $N_{\text {in }}$}

In addition to $S_{i n}$, the inlet concentration of the inorganic nitrogen $N_{\text {in }}$ may constitute a potential source of nitrogen in the reaction medium. The deficiency or excess of Nitrogen limits the development of microorganisms by preventing/inhibiting bacterial growth and causing an imbalance in their intracellular $\mathrm{pH}$. This leads to the process failure.

To get more insight into its influence, we evaluate its relationship with the concentration of the organic load. In simulations, we study the maximum tolerated value of $N_{\text {in }}$ that guarantees process stability, for the predefined range of $S_{\text {in }}\left(0<S_{\text {in }}<30\right)$ and the optimum value of the dilution rate $\left(D=0.05 \mathrm{~d}^{-1}\right)$.

We establish the operating diagram as a function of changes in $S_{i n}$ and $N_{i n}$ (Figure 14).
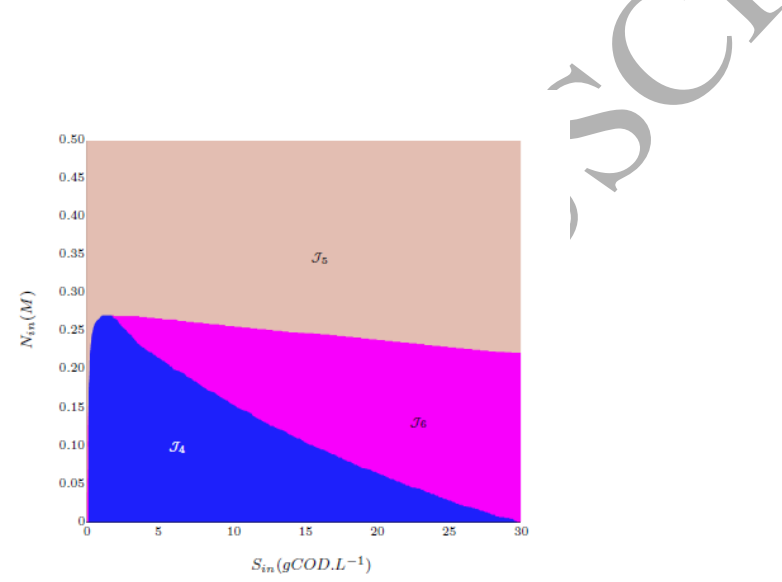

Figure 14: Operating diagram with respect to $N_{\text {in }}$ and $S_{\text {in }}$ with $D=0.05 d^{-1}$.

Table 6: Possible areas when $N_{i n}$ and $S_{i n}$ vary at $p H=7, D=0.05 d^{-1}$ : unstable (U), stable (S), unstable or does not exist $(\mathrm{X})$, equilibrium does not exist (no sign).

\begin{tabular}{lllllllllll} 
Area & $E_{1}$ & $E_{2}$ & $E_{3}^{1}$ & $E_{3}^{2}$ & $E_{4}$ & $E_{5}^{1}$ & $E_{5}^{2}$ & $E_{6}$ & $E_{7}^{1}$ & $E_{7}^{2}$ \\
\hline $\mathcal{J}_{4}$ & $U$ & $U$ & $X$ & & $X$ & $X$ & & $U$ & $S$ & \\
$\mathcal{J}_{5}$ & $U$ & $U$ & & & $U$ & $X$ & $X$ & $S$ & & \\
$\mathcal{J}_{6}$ & $U$ & $U$ & $X$ & $X$ & $U$ & $X$ & $X$ & $S$ & $S$ & $U$ \\
\hline
\end{tabular}

It is clear from Figure 14 that increasing both $N_{i n}$ and $S_{\text {in }}$ leads to bi-stability $\left(\mathcal{J}_{6}\right)$, and possibly the washout of microorganisms $\left(\mathcal{J}_{5}\right)$, due to the severe risk of 
the ammonia toxicity. Bistability or washout can, also, take place in presence of the small concentrations of $S_{i n}$ and $N_{i n}$ in the reactor. Thus, the optimal concentration of $N_{\text {in }}$ belongs to the area $\left(\mathcal{J}_{4}\right)$. It maximum value is highly dependent on the organic load: its decreases when $S_{\text {in }}$ increases.

Indeed, when $S_{\text {in }}$ reaches its maximum value and $N_{\text {in }}$ is too small the process operate in the bi-stability area $\left(\mathcal{J}_{6}\right)$ where both the equilibria $E_{6}$ and $E_{7}^{1}$ are stable. However, in the case of low concentration of $S_{i n}$ and high nitrogen inlet value, the bacteria are washed out $\left(\mathcal{J}_{5}\right)$ and only the equilibrium $E_{6}$ is stable. The washout of methanogens is due to an excess of nitrogen in the reaction medium: released from the breakdown of proteins, in the first case, and derived from the nitrogen input concentration, in the second case.

Nitrogen input has a significant influence on model behavior. A sudden rise in its value might lead to process failure even if $D$ and $S_{i n}$ belong to their optimal ranges.

\section{Discussion}

\subsection{Operating parameters acting on ammonia toxicity}

Our study highlights the qualitative properties of a model treating the digestion of a protein-rich substrate at a mesophilic temperature of $35^{\circ} \mathrm{C}$. The mathematical analysis of the model allows us to emphasize the control parameters affecting process performances. The major parameters acting on ammonia toxicity are: the organic load $\left(S_{i n}\right)$, the nitrogen input concentration $\left(N_{i n}\right)$ and the dilution rate $(D)$. An increase of the first two parameters favors a large amount of nitrogen that can lead to bi-stability. Under such conditions, a slight variation in $X_{3_{0}}$ concentration may cause process failure (Eigure 6). When the $\mathrm{pH}$ reaches a basic level, these bistabilities occur even with a small value of $S_{\text {in }}\left(\mathcal{J}_{6}, \mathcal{J}_{7}\right)$ due to the buffering capacity of the reaction medium (Figure 11). However, regardless of the system $\mathrm{pH}$, values of $S_{i n}$ and $N_{i n}$ that are too low cause the bacterial washout by nitrogen deficiency. The changes of the previous parameters greatly affect bacterial communities (Table 3 ), in particular the methanogen consortia that acts directly on biogas yield. They can be limited either by a low nitrogen concentration, or inhibited by high ammonia release or still by a large amount of VFAs in the reactor.

At a neutral $\mathrm{pH}$, using the operating diagram (Figure 6), we established that the desirable area for ideal operation of process is the area $\mathcal{J}_{4}$ where no bacterial consortia were washed out. A comparison of Figures 6 and 10 with the Figure 11 reveals that the optimum value for the $\mathrm{pH}$ is 7 . Thus, to guarantee process stability, the operator must keep the bioreactor inputs within the ranges $D[0.001-0.05] d^{-1}$, 
$S_{\text {in }}[0.03-28] g C O D . L^{-1}$, at a neutral pH. In addition, Figure 13 shows that the optimum yield of the MAD model in terms of biogas production was obtained for the following ranges $[0.001-0.05] d^{-1},[0.03-30] g C O D . L^{-1}$ of $S_{i n}$ and $D$, respectively. This was the case, when $D$ was close to $\bar{\mu}_{2}$. The maximum value of biogas production is then reached in the area of the bi-stability $\left(\mathcal{J}_{6}\right)$ where small changes to the methanogen concentration may lead to washout (boundary equilibrium $E_{6}$ ). In this case, particular attention must be paid to the $X_{3_{0}}$ concentration as well as the dilution rate value because any change on this latter can inhibit $X_{2}$ and so alter process functioning in rendering $E_{5}^{1}$ stable.

Figure 14 shows the relationship between the concentrations of $S_{i n}$ and $N_{i n}$ when D takes its optimal value for biogas production $\left(D=0.05 d^{-1}\right)$.

To ensure process stability $\left(\mathcal{J}_{4}\right)$, the ideal concentration of nitrogen inlet $\left(N_{\text {in }}\right)$ should be chosen according to the value of $S_{i n}$. In presence of a rising concentration of the organic load, $N_{\text {in }}$ value must be the smallest possible to satisfy the bacterial needs, on the one hand, and to avoid the threshold level of inhibition, on the other hand.

\subsection{MAD model limitations}

It is well known that an acidic medium in an AD bioreactor favors the formation of free VFAs, inhibitors of methanogenic bacteria. Notice however that no inhibition occured when we plotted the OD at $p H=6.5$ (Figure 10). This means that the MAD model is not able to predict realistic process behavior at low $\mathrm{pH}$. It is a limitation of the model, which it is actually due to the fact that in the study of Mairet et al. [16], the Haldane inhibition constant $\left(K_{I_{3}}=16.4 \mathrm{gCOD} . \mathrm{L}^{-1}\right)$ was too large. Bernard et al. [22] and Benyahia et al. [23] have assumed that the value of this parameter is around $256 \mathrm{mmol} . \mathrm{L}^{-1}$ and $40 \mathrm{mmol} . \mathrm{L}^{-1}$ (Table1 of [23]), which matches 0.2713 gCOD. $L^{-1}$ and 0.058 gCOD. $L^{-1}$, respectively.

To check the impact of a small value of $K_{I 3}$ parameter on process behavior, we simulated the operating diagram of the model with an intermediate value of $K_{I 3}$, about $0.1 \mathrm{gCOD} \cdot \mathrm{L}^{-1}$. 


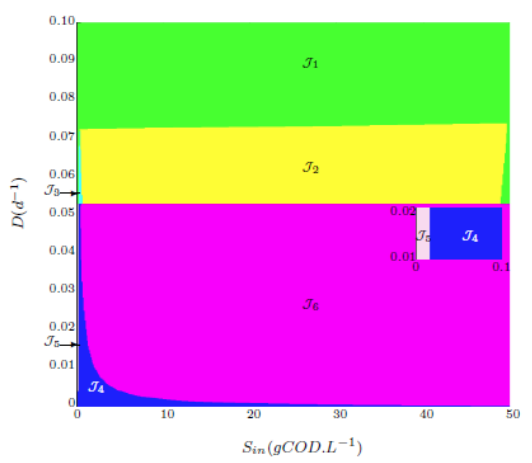

Figure 15: Operating diagram showing equilibria behavior as a function of $D$ and $S_{\text {in }}$ at $p H=6.5$, $N_{\text {in }}=0.011 M, K_{I 3}=0.1$. The area $\mathcal{J}_{5}$ is too small in the OD because it appears for only small values of $S_{i n}$.

Table 7: Existence and nature of equilibria as a function of $S_{\text {in }}$ and $D$ at $p H=6.5, N_{\text {in }}=0.011 M$, $K_{I 3}=0.1$ : unstable $(\mathrm{U})$, stable $(\mathrm{S})$, unstable or does not exist $(\mathrm{X})$, equilibria does not exist (no $\operatorname{sign})$.

\begin{tabular}{|c|c|c|c|c|c|c|c|c|c|c|}
\hline Area & $E_{1}$ & $E_{2}$ & $E_{3}^{1}$ & & & $E_{5}^{1}$ & $E_{5}^{2}$ & $E_{6}$ & $E_{7}^{1}$ & $E_{7}^{2}$ \\
\hline $\mathcal{J}_{1}$ & $U$ & & & & & & & & & \\
\hline $\mathcal{J}_{2}$ & $U$ & & & & & $S$ & $U$ & & & \\
\hline $\mathcal{J}_{3}$ & $U$ & & & & & $S$ & & & & \\
\hline $\mathcal{J}_{4}$ & $U$ & & & & $U$ & $U$ & & $U$ & $S$ & \\
\hline $\mathcal{J}_{5}$ & $U$ & & & & $U$ & $X$ & $X$ & $S$ & & \\
\hline $\mathcal{J}_{6}$ & & & & $X$ & $U$ & $X$ & $X$ & $S$ & $S$ & $U$ \\
\hline
\end{tabular}

Figure 15 shows that, at an acidic $\mathrm{pH}$, the MAD model becomes more sensitive to high VFAs concentrations with this value of $K_{I 3}$. The appropriate operational region for process operation $\left(\mathcal{J}_{4}\right)$ narrows and the instability is reached at a value of $D$ and $S_{i n}$ ensuring the stability while $K_{I 3}=16.4 \mathrm{gCOD} \cdot \mathrm{L}^{-1}$. This parameter plays a significant role when the process operates at a low $\mathrm{pH}$ where the formation of un-ionized VFAs is considerable. 


\section{Properties of the ADM1m model in view of the properties of MAD model}

Mairet et al. [16] showed that the MAD model presents the same dynamic behavior as a modified ADM1 model [17]: ADM1 adapted to the AD of microalgae, called hereafter ADM1m. Investigating the qualitative properties of such a complex model give a general insight into process behavior, useful in the case of operation and control of the production of biogas.

These properties can be investigated either by simulations requiring powerful numerical tools [24], or by the study of a reduced ADM1m model. Based on the equivalence of the ADM1m model and the MAD model, we try, to investigate the qualitative and quantitative properties of the ADM1m model using the findings on the MAD model properties presented above.

\subsection{Qualitative properties of the ADM1m model}

From the OD of the MAD model (Figure 6) we can observe that, for a fixed value of $S_{\text {in }}=2 \mathrm{gCOD} . \mathrm{L}^{-1}$, the progressive increase of $D$ leads to the emergence of areas $\mathcal{J}_{4}, \mathcal{J}_{3}, \mathcal{J}_{1}, \mathcal{J}_{0}$.

The transition from the operational zone $\mathcal{J}_{4}$ to $\mathcal{J}_{3}, \mathcal{J}_{1}$ and $\mathcal{J}_{0}$ involves, respectively, the washout of the bacteria $X_{2}, X_{3}$ and $X_{1}$. The total washout of microorganisms is then indicated by the area $\mathcal{J}_{0}$, obtained for large values of the dilution rate. However, we can see from the same figure that small values of $S_{i n}$ induce bacterial washout by nitrogen while, higher values of $S_{i n}$ led to bi-stability, even to washout area when $K_{I 3}$ is too low (Figure 15).

Thus, the washout can be caused either by a high dilution rate, a low organic load or by an organic overload.

Compared to the original ADM1, the simulations of the ADM1m model show a shift in the amount of various intermediate products, mainly the inorganic nitrogen concentration [17]. This is due to the different hydrolysis kinetics considered by the two models: a first-order kinetic and a Contois kinetic are assigned for the first and second models, respectively. Fekih-Salem et al. [25] demonstrate that, in the case of the 'Anaerobic digestion models', the change in the hydrolysis rate may act only on the amount of soluble organic matter. It assumes, then, that the qualitative properties/are not affected.

Hence, to verify our results and enhance scope of their validity, we tried to compare the qualitative properties of the ADM1m model with those of the orginal ADM1 numerically studied by Bornhöft et al. [24]. The authors stressed that the washout of the ADM1 model can occur either by: i) an increase in the dilution rate; ii) a 
decrease of substrate concentration (due to famine); or iii) an increase of input substrate concentration (leading to inhibition).

All these properties of the ADM1 model correspond very well to the properties of the ADM1m model [24].

\subsection{Quantitative properties of the ADM1m model}

As well as qualitative properties, quantitative properties of the ADM1m model can also be revealed from the equilibria calculation of the MAD model. Khedim et al. [26], established equivalence relationships between the MAD and ADM1m models using the states-variables association method proposed in [27]. These relations are simple algebraic equations where each variable of the MAD model is defined as a combination of variables of the ADM1m model (Table 8).

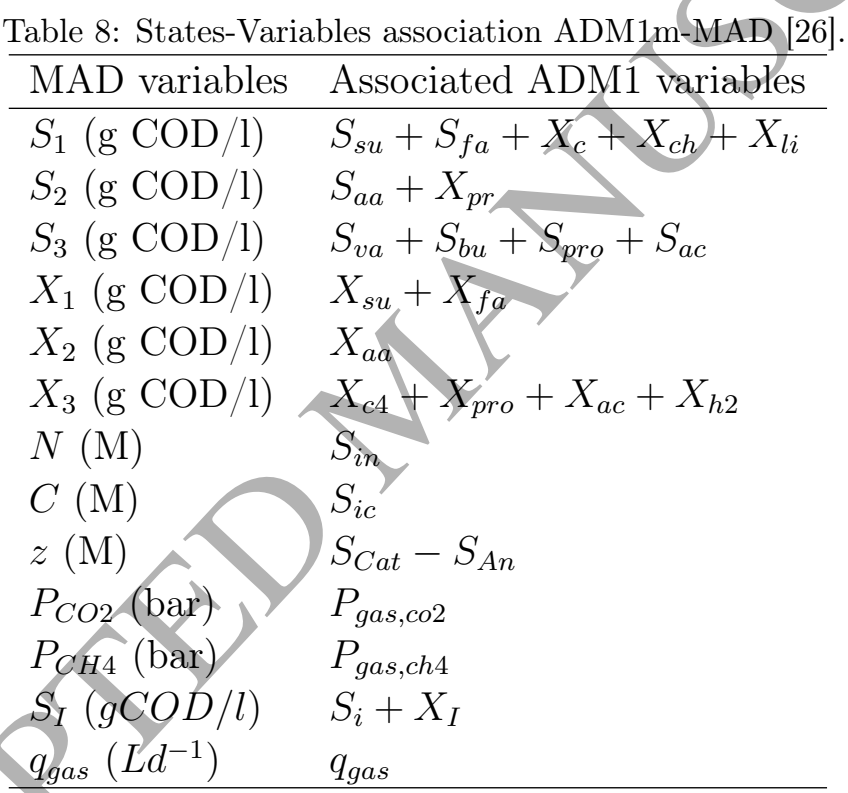

Based on these combinations, we have evaluated the equilibrium value of the MAD and ADM1m models.

We apply the same dilution rates and organic loads to both models. Then, for each value of $D$ and $S_{i n}$, we calculate the value of the stable equilibrium in the corresponding area. In the case of bi-stability $\left(\mathcal{J}_{6}\right.$ or $\left.\mathcal{J}_{2}\right)$, we represent the interior point (positive equilibrium excluding washout). Calculation results are reported in Table 9.)

Table 9 shows a good correspondence between the equilibrium values of the MAD 
Table 9: Equilibria values of ADM1m-MAD models in each area.

\begin{tabular}{llllllllll}
\hline Area & models & Dilution rate / organic load values & $S_{1}$ & $S_{2}$ & $S_{3}$ & $X_{1}$ & $X_{2}$ & $X_{3}$ & $N$ \\
\hline $\mathcal{J}_{0}$ & ADM1m & $D=0.35, S_{\text {in }}=15$ & 4.1602 & 6.1747 & 0 & 0 & 0 & 0 & 0.0109 \\
& MAD & 4.5 & 6 & 0 & 0 & 0 & 0 & 0.0110 \\
\hline $\mathcal{J}_{1}$ & ADM1m & $D=0.2, S_{\text {in }}=15$ & 2.4625 & 6.2185 & 0.3642 & 0.1494 & 0 & 0.0718 & 0.0095 \\
& MAD & & 1.1435 & 6 & 3.08 & 0.2685 & 0 & 0 & 0.0093 \\
\hline $\mathcal{J}_{2}$ & ADM1m & $D=0.12, S_{\text {in }}=28$ & 1.4806 & 11.6867 & 0.1591 & 0.4376 & 0 & 0.2947 & 0.0061 \\
& MAD & & 0.8545 & 11.2000 & 0.1653 & 0.6036 & 0 & 0.3388 & 0.0051 \\
\hline $\mathcal{J}_{3}$ & ADM1m & $D=0.07, S_{\text {in }}=15$ & 0.3526 & 6.3002 & 0.0866 & 0.2341 & 0 & 0.1600 & 0.0082 \\
& MAD & & 0.2211 & 6 & 0.0217 & 0.3423 & 0 & 0.1957 & 0.0076 \\
\hline $\mathcal{J}_{4}$ & ADM1m & $D=0.025, S_{\text {in }}=15$ & 0.1574 & 0.1523 & 0.0553 & 0.1807 & 0.2843 & 0.4081 & 0.0479 \\
& MAD & & 0.0684 & 0.0328 & 0.0057 & 0.3545 & 0.6557 & 0.4691 & 0.0413 \\
\hline $\mathcal{J}_{6}$ & ADM1m & $D=0.0491, S_{\text {in }}=29.5$ & 0.4144 & 1.1963 & 0.1161 & 0.4432 & 0.6503 & 0.9537 & 0.0759 \\
& MAD & & 0.2845 & 0.8315 & 0.0191 & 0.6852 & 1.205 & 0.8812 & 0.0663 \\
\hline
\end{tabular}

model and those of the ADM1m model. Slight differences between the values of some variables may be due to the fact that bacterial death is not accounted for the MAD model.

However, a significant offset in the VFA concentration can be observed in most regions. These gaps in the results can be explained by the fact that the MAD model considers only the acetic acid while the modified ADM1 model takes into account also the propionic, valeric, butyric, acetic acids. The high value of the Haldane inhibition constant $K_{I 3}$ considered in the MAD model can also be another cause of such mismatches. These results validate, on one hand, the theoretical results obtained by the mathematical study of the MAD model and stress its ability - with a modified Haldane inhibition constant - to predict the behavior of a more complex model such as the ADM1m model.

Thus, ADM1m can be controlled via the study of the MAD model, which appears to be a good candidate for synthesizing control laws.

\section{Conclusion}

This paper presents the mathematical analysis of a Microalgae Anaerobic Digestion (MAD) model. The qualitative properties of the model were compared with those of the original and modified ADM1 models. Simulation results show the key role of control parameters in process behavior as well as the strong effect of $\mathrm{pH}$ on the digestion of protein-rich substrates. On the one hand, these results enable us to identify the ideal conditions for the operation of such digestion. On the other, they give an insight into the behavior of the modified ADM1 solutions. Thus, they should be very helpful for controlling AD processes treating protein-rich substrates. 
Perspectives of this work include the extension of the present results to the case where the $\mathrm{pH}$ is not constant, stressing the qualitative properties of the model in such cases. This will be useful for knowing how to proceed under the different pHs of the system as well as for a wide range of organic load and dilution rates.

\section{Appendix A. Proof of proposition 1}

Proof. An equilibrium point $\xi^{*}=\left(S_{1}^{*}, S_{2}^{*}, S_{3}^{*}, X_{1}^{*}, X_{2}^{*}, X_{3}^{*}, N^{*}\right)$ of the system must be a solution of (19a-19g) with the left-hand side equal to zero. From equation (19d), we have:

- $X_{1}^{*}=0$; hence, using (19a), one obtains $S_{1}^{*}=\beta_{1} S_{\text {in }}$

- or $S_{1}^{*}$ and $X_{1}^{*}$ must satisfy equation $\mu_{1}\left(S_{1}, X_{1}\right)=D$; hence, using (19a), one gets $S_{1}^{*}$ and $X_{1}^{*}$ together as a solution of $(20 \mathrm{a}-20 \mathrm{~b})$. Therefore $S_{1}^{*}$ and $X_{1}^{*}$ are given by (25) and (24), respectively.

Similarly, from equation (19e) it is deduced that:

- $X_{2}^{*}=0$; hence, using (19b), one obtains $S_{2}^{*}=\beta_{2} S_{\text {in }}$

- or $S_{2}^{*}$ and $X_{2}^{*}$ must satisfy equation $\mu_{2}\left(S_{2}, X_{2}\right)=D$; hence, using (19b), one obtains $S_{2}^{*}$ and $X_{2}^{*}$ as a solution of $(21 \mathrm{a}-21 \mathrm{~b})$. Therefore, $S_{2}^{*}$ and $X_{2}^{*}$ are given by $(28)$ and $(27)$, respectively.

On the other hand, from equation (19f) it is deduced that:

- $X_{3}^{*}=0$; hence, using (19c) and (19g), one obtains $S_{3}^{*}$ and $N^{*}$ with respect of $S_{1}^{*}, X_{1}^{*}, S_{2}^{*}$ and $X_{2}^{*}$

- or $S_{3}^{*}$ and $N^{*}$ must satisfy equation $\mu_{3}\left(S_{3}, N\right)=D$; hence, using (19c) and (19g), one obtains $S_{3}^{*}, X_{3}^{*}$ and $N^{*}$ as a solution of (22a-22c). Therefore, $X_{3}^{*}$ and $N^{*}$ are given by (29) and (30), respectively, where $S_{3}^{*}$ is a solution of equation (32) satisfying the condition $S_{3}^{*}<S_{3 i n}^{*}$.

Eight cases must be distinguished, as shown in Table 1 . The 8th case corresponding to $X_{1}=0, X_{2}=0, X_{3} \neq 0$ cannot occur since, according to (19f), we would have $\mu_{3}=D$ and hence, using (19c) we will obtain

$$
D S_{3}^{*}+\alpha_{9} X_{3}^{*}=0
$$

which is impossible (positivity property of biological systems is not fulfilled). The rest of the proof is illustrated in Figure 5. 


\section{Appendix B. Analytical calculation of equilibria nature (c.f 2.3)}

When we consider $\xi=\left[S_{1}, X_{1}, S_{2}, X_{2}, S_{3}, X_{3}, N\right]^{T}$, the state vector of the model MAD, the Jacobian matrix will be described as a block-diagonal structure:

$$
J=\left(\begin{array}{ccc}
A & 0_{2 * 2} & 0_{2 * 3} \\
0_{2 * 2} & B & 0_{2 * 3} \\
E & F & C
\end{array}\right)
$$

where

$$
\begin{gathered}
A=\left(\begin{array}{cc}
-D-\alpha_{1} X_{1} \mu_{1 S}^{\prime} & -X_{1} \alpha_{1} \mu_{1 X}^{\prime}-\alpha_{1} \mu_{1}\left(S_{1}^{*}, X_{1}^{*}\right) \\
X_{1} \mu_{1 S}^{\prime} & \mu_{1}\left(S_{1}^{*}, X_{1}^{*}\right)-D+X_{1} \mu_{1 X}^{\prime}
\end{array}\right) \\
B=\left(\begin{array}{cc}
-D-\alpha_{5} X_{2} \mu_{2 S}^{\prime} & -X_{2} \alpha_{5} \mu_{2 X}^{\prime}-\alpha_{5} \mu_{2}\left(S_{2}^{*}, X_{2}^{*}\right) \\
X_{2} \mu_{2 S}^{\prime} & \mu_{2}\left(S_{2}^{*}, X_{2}^{*}\right)-D+X_{2} \mu_{2 X}^{\prime}
\end{array}\right), \\
E=\left(\begin{array}{cc}
\alpha_{3} X_{1} \mu_{1 S}^{\prime} & \alpha_{3} X_{1} \mu_{1 X}^{\prime}+\alpha_{3} \mu_{1}\left(S_{1}^{*}, X_{1}^{*}\right) \\
0 & 0 \\
-\alpha_{2} X_{1} \mu_{1 S}^{\prime} & -\alpha_{2} \mu_{1}\left(S_{1}^{*}, X_{1}^{*}\right)-\alpha_{2} X_{1} \mu_{1 X}^{\prime}
\end{array}\right), \\
F=\left(\begin{array}{cc}
\alpha_{6} X_{2} \mu_{2 S}^{\prime} & \alpha_{6} X_{2} \mu_{2 X}^{\prime}+\alpha_{6} \mu_{2}\left(S_{2}^{*}, X_{2}^{*}\right) \\
0 & \alpha_{7} \mu_{2}\left(S_{2}^{*}, X_{2}^{*}\right)+\alpha_{7} X_{2} \mu_{2 X}^{\prime}
\end{array}\right), \\
\alpha_{7} X_{2} \mu_{2 S}^{\prime} \\
C \\
C=\left(\begin{array}{ccc}
C 11 & C 12 & C 13 \\
C 21 & C 22 & C 23 \\
C 31 & C 32 & C 33
\end{array}\right) .
\end{gathered}
$$

with

$$
\begin{aligned}
& C 11=-D-\alpha_{9} X_{3} \mu_{3 S}^{\prime} \quad, \quad C 12=-\alpha_{9} \mu_{3} \quad, \quad C 13=-\alpha_{9} X_{3} \mu_{3 N}^{\prime} \\
& C 21=X_{3} \mu_{3 S}^{\prime} \quad, C 22=-D+\mu_{3} \quad, \quad C 23=X_{3} \mu_{3 N}^{\prime} \\
& C 31=-\alpha_{10} X_{3} \mu_{3 S}^{\prime} \quad, C 32=-\alpha_{10} \mu_{3} \quad, \quad C 33=-D-X_{3} \alpha_{10} \mu_{3 N}^{\prime}
\end{aligned}
$$

and

$$
\mu_{1 S}^{\prime}=\frac{\partial \mu_{1}\left(S_{1}^{*}, X_{1}^{*}\right)}{\partial S_{1}}, \quad \mu_{1 X}^{\prime}=\frac{\partial \mu_{1}\left(S_{1}^{*}, X_{1}^{*}\right)}{\partial X_{1}}, \quad \mu_{2 S}^{\prime}=\frac{\partial \mu_{2}\left(S_{2}^{*}, X_{2}^{*}\right)}{\partial S_{2}}
$$




$$
\mu_{2 X}^{\prime}=\frac{\partial \mu_{2}\left(S_{2}^{*}, X_{2}^{*}\right)}{\partial X_{2}}, \quad \mu_{3 S}^{\prime}=\frac{\partial \mu_{3}\left(S_{3}^{*}, N^{*}\right)}{\partial S_{3}}, \quad \mu_{3 N}^{\prime}=\frac{\partial \mu_{3}\left(S_{3}^{*}, N^{*}\right)}{\partial N}
$$

The eigenvalues of the Jacobian matrix are the eigenvalues of matrices A, B and C. Since A and B are square matrices, we can calculate their trace and determinant. To guarantee the system stability: traces must be negatives $(\operatorname{tr}($ matrix $)<0)$ and determinants must be positives ( $\operatorname{det}($ matrix $)>0$ ).

The stability of the matrix $\mathrm{C}$ can be tested using the Routh-Hurwitz criterion, in particular in the case of the equilibria $E_{5}^{i}$ and $E_{7}^{i}$. For example

- The calculation of Jacobian matrix at the equilibrium $E_{1}$ gives:

$$
\begin{gathered}
A=\left(\begin{array}{cc}
-D & -a_{1} \mu_{1}\left(\beta_{1} S_{i n}, 0\right) \\
0 & \mu_{1}\left(\beta_{1} S_{i n}, 0\right)-D
\end{array}\right), \\
B=\left(\begin{array}{cc}
-D & -a_{5} \mu_{2}\left(\beta_{2} S_{i n}, 0\right) \\
0 & \mu_{2}\left(\beta_{2} S_{i n}, 0\right)-D
\end{array}\right) \\
C=\left(\begin{array}{ccc}
-D & -\alpha_{9} \mu_{3}\left(0, N_{i n}\right) & 0 \\
0 & -D+\mu_{3}\left(0, N_{i n}\right) & 0 \\
0 & -a 10 \mu_{3}\left(0, N_{i n}\right) & -D
\end{array}\right) .
\end{gathered}
$$

The eigenvalues of $\mathrm{A}$ and $\mathrm{B}$ are negative (i.e. $\operatorname{tr}(A)<0, \operatorname{tr}(B)<0)$ and $(\operatorname{det}(A)>0, \operatorname{det}(B)>0)$ if, and only if, $\mu_{1}\left(\beta_{1} S_{i n}, 0\right)<D, \mu_{2}\left(\beta_{2} S_{i n}, 0\right)<D$, respectively.

Since the matrix $C$ is block-triangular, its eigenvalues are $-D$ and the eigenvalues of the second order matrix in the upper left corner. Since, this second order matrix is an upper triangular matrix, its eigenvalues are on the diagonal, i.e. $-D$ and $-D+\mu_{3}\left(0, N_{i n}\right)$. Thus, $C$ is stable if, and only if, $\mu_{3}\left(0, N_{i n}\right)<D$. The stability of the matrix $C$ can also be investigated using the Routh-Hurwitz criterion where the characteristic polynomial is defined by:

$$
P_{E_{1}}=\Lambda^{3}+\left(-\mu_{3}\left(0, N_{i n}\right)+3 D\right) \Lambda^{2}+\left(3 D^{2}-2 D \mu_{3}\left(0, N_{i n}\right)\right) \Lambda-D^{2} \mu_{3}\left(0, N_{i n}\right)+D^{3}
$$

Thus, the Routh array is

\begin{tabular}{ll|lll}
$\Lambda^{3}$ & 1 & $3 D^{2}-2 D \mu_{3}\left(0, N_{i n}\right)$ & 0 \\
$\Lambda^{2}$ & $-\mu_{3}\left(0, N_{i n}\right)+3 D$ & $-D^{2} \mu_{3}\left(0, N_{i n}\right)+D^{3}$ & 0 \\
$\Lambda^{1}$ & $\operatorname{cst1}$ & 0 & 0 \\
$\Lambda^{0}$ & $\operatorname{cst2}$ & 0 & 0
\end{tabular}


with

$$
\begin{aligned}
\operatorname{cst} 1= & \frac{1}{-\mu_{3}\left(0, N_{\text {in }}\right)+3 D}\left[\left(-\mu_{3}\left(0, N_{\text {in }}\right)+3 D\right)\left(3 D^{2}-2 D \mu_{3}\left(0, N_{\text {in }}\right)\right)-\left(-\mu_{3}\left(0, N_{\text {in }}\right)\right.\right. \\
& \left.\left.D^{2}+D^{3}\right)\right] \\
\text { cst } 2= & -D^{2} \mu_{3}\left(0, N_{\text {in }}\right)+D^{3}
\end{aligned}
$$

The Routh-Hurwitz criterion require that all the terms of the first column of array must have the same sign. Since the first term of the first column is positive then all the subsequent terms must be positive, i.e. $-\mu_{3}\left(0, N_{\text {in }}\right)+3 D$, cst 1 and $c s t 2$, to fullfill the stability condition. It yields that the necessar condition to garantee that the eigenvalues of the matrix $\mathrm{C}$ are negative is $\mu_{3}\left(0, N_{\text {in }}\right)<D$. Hence, if $S_{1}^{*}<\beta_{1} S_{i n}, S_{2}^{*}<\beta_{2} S_{\text {in }}$ and $S_{3}^{*, i}<S_{3 i n}^{*}$, the equilibrium $E_{1}$ is locally asymptotically stable. This equilibrium is unstable if $S_{1}^{*}>\beta_{1} S_{i n}, S_{2}^{*}>\beta_{2} S_{i n}$ or $S_{3}^{*, i}>S_{3 i n}^{*}$.

- The assessment of the Jacobian matrix around the equilibrium $E_{4}$ gives:

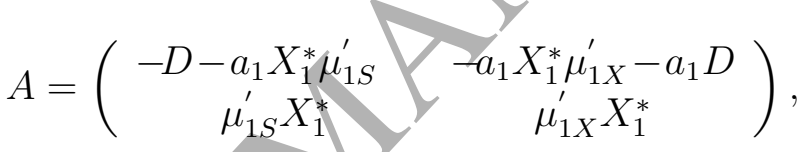

$$
\begin{aligned}
& B=\left(\begin{array}{cc}
-D y & -a_{5} \mu_{2}\left(\beta_{2} S_{i n}, 0\right) \\
0 & \mu_{2}\left(\beta_{2} S_{i n}, 0\right)-D
\end{array}\right), \\
& C=\left(\begin{array}{ccc}
-D & -\alpha_{9} \mu_{3}\left(S_{3 i n, 1}^{*}, N_{i n, 1}^{*}\right) & 0 \\
0 & \mu_{3}\left(S_{3 i n, 1}^{*}, N_{i n, 1}^{*}\right)-D & 0 \\
0 & -\alpha_{10} \mu_{3}\left(S_{3 i n, 1}^{*}, N_{i n, 1}^{*}\right) & -D
\end{array}\right)
\end{aligned}
$$

The eigenvalues of the matrix $B$ has a negative real part if, and only if, $\mu_{2}\left(\beta_{2} S_{i n}, 0\right)<D$. We obtain for matrix A :

$$
\begin{gathered}
\operatorname{tr}(A)=-D-\alpha_{1} X_{1}^{*} \mu_{1 S}^{\prime}+\mu_{1 X}^{\prime} X_{1}^{*} \\
\operatorname{det}(A)=D X_{1}^{*}\left(-\mu_{1 X}^{\prime}+\alpha_{1} \mu_{1 S}^{\prime}\right)
\end{gathered}
$$

The eigenvalues of the matrix A are negatives if, and only if,

$$
\alpha_{1} \mu_{1 S}^{\prime}>\mu_{1 X}^{\prime}
$$


In this case, the characteristic polynomial of the matrix $\mathrm{C}$ is:

$$
\begin{aligned}
P_{E_{4}}= & \Lambda^{3}+\left(3 D-\mu_{3}\left(S_{3 i n, 1}^{*}, N_{i n, 1}^{*}\right)\right) \Lambda^{2}+\left(3 D^{2}-2 D \mu_{3}\left(S_{3 i n, 1}^{*}, N_{i n, 1}^{*}\right) \Lambda+D^{2}(D\right. \\
& \left.-\mu_{3}\left(S_{3 i n, 1}^{*}, N_{i n, 1}^{*}\right)\right)
\end{aligned}
$$

Using the Routh-Hurwitz criterion, we can deduce that the matrix $C$ is stable if, and only if, $\mu_{3}\left(S_{3 i n, 1}^{*}, N_{i n, 1}^{*}\right)<D$. This can also be deduced easily from the structure of the matrix $\mathrm{C}$ whose eigenvalues are simply the values on the diagonal, i. e. $-D, \mu_{3}\left(S_{3 i n, 1}^{*}, N_{i n, 1}^{*}\right)-D$ and $-D$. The matrix is stable if, and only if, $\mu_{3}\left(S_{3 i n, 1}^{*}, N_{i n, 1}^{*}\right)<D$.

- We obtain from calculation of the Jacobian matrix around the equilibria $E_{5}^{i}$ $(i=1,2)$, the following submatrices:

$$
\begin{gathered}
A=\left(\begin{array}{cc}
-D-a_{1} X_{1}^{*} \mu_{1 S}^{\prime} & -a_{1} X_{1}^{*} \mu_{1 X}^{\prime}-a_{1} D \\
\mu_{1 S}^{\prime} X_{1}^{*} & \mu_{1 X}^{\prime} X_{1}^{*}
\end{array}\right), \\
B=\left(\begin{array}{cc}
-D & -a_{5} \mu_{2}\left(\beta_{2} S_{i n}, 0\right) \\
0 & \mu_{2}\left(\beta_{2} S_{i n}, 0\right)-D
\end{array}\right), \\
C=\left(\begin{array}{ccc}
C 11_{E_{5}^{i}} & C 12_{E_{5}^{i}} & C 13_{E_{5}^{i}} \\
C 21_{E_{5}^{i}} & C 22_{E_{5}^{i}} & C 23_{E_{5}^{i}} \\
C 31_{E_{5}^{i}} & C 32_{E_{5}^{i}} & C 33_{E_{5}^{i}}^{i}
\end{array}\right) .
\end{gathered}
$$

with

$$
\begin{aligned}
C 11_{E_{5}^{i}} & =-D-\left(S_{3 i n, 1}^{*}-S_{3}^{*}\right) \mu_{3 S}^{\prime} \\
C 12_{E_{5}^{i}} & =-\alpha_{9} D \\
C 13_{E_{5}^{i}} & =-\left(S_{3 i n, 1}^{*}-S_{3}^{*}\right) \mu_{3 N}^{\prime} \\
C 21_{E_{5}^{i}} & =\frac{\left(S_{3 i n, 1}^{*}-S_{3}^{*}\right)}{\alpha_{9}} \mu_{3 S}^{\prime} \\
C 22_{E_{5}^{i}} & =0 \\
C 23_{E_{5}^{i}} & =\frac{\left(S_{3 i n, 1}^{*}-S_{3}^{*}\right)}{\alpha_{9}} \mu_{3 N}^{\prime}
\end{aligned}
$$




$$
\begin{aligned}
C 31_{E_{5}^{i}} & =-a_{10} \frac{\left(S_{3 i n, 1}^{*}-S_{3}^{*}\right)}{\alpha_{9}} \mu_{3 S}^{\prime} \\
C 32_{E_{5}^{i}} & =-\alpha_{10} D \\
C 33_{E_{5}^{i}} & =-D-a_{10} \frac{\left(S_{3 i n, 1}^{*}-S_{3}^{*}\right)}{\alpha_{9}} \mu_{3 N}^{\prime}
\end{aligned}
$$

The eigenvalues of the matrix $\mathrm{B}$ have a negative real part if, and only if, $\mu_{2}\left(\beta_{2} S_{\text {in }}, 0\right)<D$. We obtain for matrix A:

$$
\begin{gathered}
\operatorname{tr}(A)=-D-\alpha_{1} X_{1}^{*} \mu_{1 S}^{\prime}+\mu_{1 X}^{\prime} X_{1}^{*} \\
\operatorname{det}(A)=D X_{1}^{*}\left(-\mu_{1 X}^{\prime}+\alpha_{1} \mu_{1 S}^{\prime}\right)
\end{gathered}
$$

The eigenvalues of $\mathrm{A}$ are negatives if, and only if,

$$
\alpha_{1} \mu_{1 S}^{\prime}>\mu_{1 X}^{\prime}
$$

However, the stability of matrix $C$ can be checked using the Routh-Hurwitz criterion, where the characteristic polynomial is:

$$
\begin{aligned}
P_{E_{5}^{i}}= & \Lambda^{3}+\frac{1}{\alpha_{9}}\left(2 \alpha_{9} D+\alpha_{10} \mu_{3 N}^{\prime} S_{3 i n}^{*}-\alpha_{10} \mu_{3 N}^{\prime} S_{3}^{*}-\alpha_{9} \mu_{3 S}^{\prime} S_{3}^{*}+\alpha_{9} \mu_{3 S}^{\prime} S_{3 i n}^{*}\right) \Lambda^{2}+\frac{1}{\alpha_{9}} \\
& \left(2 D S_{3 i n}^{*} \alpha_{10} \mu_{3 N}^{\prime}-2 S_{3}^{*} \alpha_{10} D \mu_{3 N}^{\prime}+2 \mu_{3 S}^{\prime} D S_{3 i n}^{*} \alpha_{9}-2 \mu_{3 S}^{\prime} S_{3}^{*} \alpha_{9} D+\alpha_{9} D^{2}\right) \Lambda+ \\
& \frac{1}{\alpha_{9}}\left(\mu_{3 S}^{\prime} D^{2} S_{3 i n}^{*} \alpha_{9}-\mu_{3 N}^{\prime} \alpha_{10} D^{2} S_{3}^{*}-\mu_{3 S}^{\prime} D^{2} S_{3}^{*} \alpha_{9}+\mu_{3 N}^{\prime} \alpha_{10} D^{2} S_{3 i n}^{*}\right)
\end{aligned}
$$

The stability is guaranteed when all the coefficients of the first column of Routh matrix retain the same sign. In this case, the conditions of stability can't be easily deduced.

- The calculation of Jacobian matrix at the equilibrium $E_{6}$, gives:

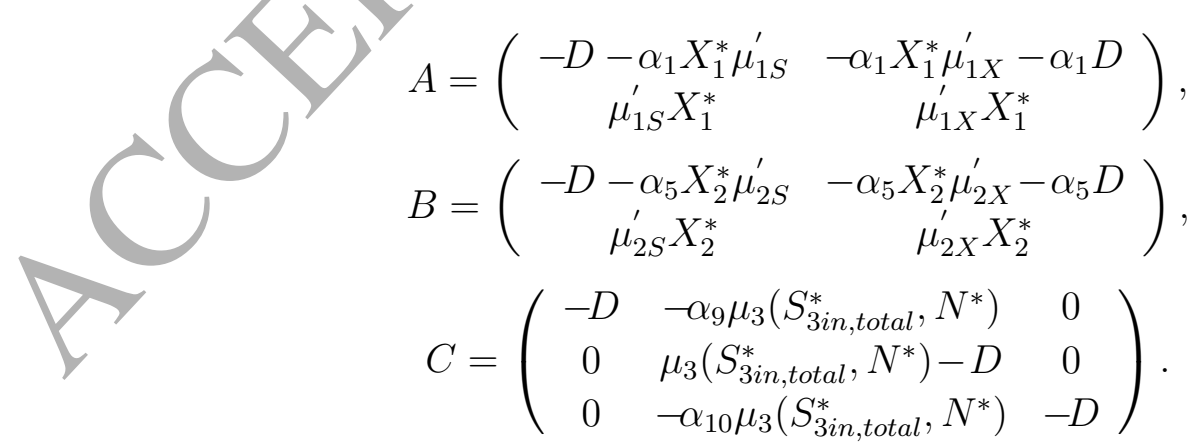


We obtain for matrix A

$$
\begin{gathered}
\operatorname{tr}(A)=-D-\alpha_{1} X_{1}^{*} \mu_{1 S}^{\prime}+\mu_{1 X}^{\prime} X_{1}^{*} \\
\operatorname{det}(A)=D X_{1}^{*}\left(-\mu_{1 X}^{\prime}+\alpha_{1} \mu_{1 S}^{\prime}\right)
\end{gathered}
$$

The eigenvalues of the matrix $\mathrm{A}$ are negative if, and only if,

$$
\alpha_{1} \mu_{1 S}^{\prime}>\mu_{1 X}^{\prime}
$$

We have for matrix B

$$
\begin{gathered}
\operatorname{tr}(B)=-D-\alpha_{5} X_{2}^{*} \mu_{2 S}^{\prime}+\mu_{2 X}^{\prime} X_{2}^{*} \\
\operatorname{det}(B)=X_{2}^{*} D\left(-\mu_{2 X}^{\prime}+\alpha_{5} \mu_{2 S}^{\prime}\right)
\end{gathered}
$$

The eigenvalues of the matrix $B$ are negative if, and only if,

The characteristic polynomial of matrix $\mathrm{C}$ is:

$$
\begin{aligned}
P_{E_{6}}= & \Lambda^{3}+\left(3 D-\mu_{3}\left(S_{3 i n, t o t a l}^{*}, N^{*}\right)\right) \Lambda^{2}+\left(3 D^{2}-2 D \mu_{3}\left(S_{3 i n, t o t a l}^{*}, N^{*}\right)\right) \Lambda+D^{2} \\
& \left(D-\mu_{3}\left(S_{3 i n, t o t a l}^{*}, N^{*}\right)\right)
\end{aligned}
$$

Using the Routh-Hurwitz criterion, we can deduce that the matrix $C$ is stable if, and only if, $D>\mu_{3}\left(S_{3 i n, t o t a l}^{*}, N^{*}\right)$. This condition guarantees that all the coefficients of the first column of Routh table retain the same sign. This can also be deduced evidently from the block-triangular form of the matrix $C$

- The Jacobian matrix calculation around the equilibria $E_{7}^{i}(i=1,2)$, gives:

$$
\begin{aligned}
& \text { ( } A=\left(\begin{array}{cc}
-D-\alpha_{1} X_{1}^{*} \mu_{1 S}^{\prime} & -\alpha_{1} X_{1}^{*} \mu_{1 X}^{\prime}-\alpha_{1} D \\
\mu_{1 S}^{\prime} X_{1}^{*} & \mu_{1 X}^{\prime} X_{1}^{*}
\end{array}\right) \text {, } \\
& B=\left(\begin{array}{cc}
-D-\alpha_{5} X_{2}^{*} \mu_{2 S}^{\prime} & -\alpha_{5} X_{2}^{*} \mu_{2 X}^{\prime}-\alpha_{5} D \\
\mu_{2 S}^{\prime} X_{2}^{*} & \mu_{2 X}^{\prime} X_{2}^{*}
\end{array}\right), \\
& C=\left(\begin{array}{lll}
C 11_{E_{7}^{i}} & C 12_{E_{7}^{i}} & C 13_{E_{7}^{i}} \\
C 21_{E_{7}^{i}} & C 22_{E_{7}^{i}} & C 23_{E_{7}^{i}} \\
C 31_{E_{7}^{i}} & C 32_{E_{7}^{i}} & C 33_{E_{7}^{i}}
\end{array}\right)
\end{aligned}
$$


with

$$
\begin{aligned}
& C 11_{E_{7}^{i}}=-D-\left(S_{3 i n, t o t a l}^{*}-S_{3}^{*}\right) \mu_{3 S}^{\prime} \\
& C 12_{E_{7}^{i}}=-\alpha_{9} D \\
& C 13_{E_{7}^{i}}=-\left(S_{3 \text { in,total }}^{*}-S_{3}^{*}\right) \mu_{3 N}^{\prime} \\
& C 21_{E_{7}^{i}}=\frac{\mu_{3 S}^{\prime}\left(S_{3 i n, t o t a l}^{*}-S_{3}^{*}\right)}{\alpha_{9}} \\
& C 22_{E_{7}^{i}}=0 \\
& C 23_{E_{7}^{i}}=\frac{\mu_{3 N}^{\prime}\left(S_{3 i n, t o t a l}^{*}-S_{3}^{*}\right)}{\alpha_{9}} \\
& C 31_{E_{7}^{i}}=-\frac{\alpha_{10}\left(S_{3 i n, t o t a l}^{*}-S_{3}^{*}\right) \mu_{3 S}^{\prime}}{\alpha_{9}} \\
& C 32_{E_{7}^{i}}=-\alpha_{10} D \\
& C 33_{E_{7}^{i}}=-D-\frac{\alpha_{10}\left(S_{3 i n, t o t a l}^{*}-S_{3}^{*}\right) \mu_{3 N}^{\prime}}{\alpha_{9}}
\end{aligned}
$$

For matrix A, we have

$$
\begin{gathered}
\operatorname{tr}(A)=-D-\alpha_{1} X_{1}^{*} \mu_{1 S}^{\prime}+\mu_{1 X}^{\prime} X_{1}^{*} \\
\operatorname{det}(A)=D X_{1}^{*}\left(-\mu_{1 X}^{\prime}+\alpha_{1} \mu_{1 S}^{\prime}\right)
\end{gathered}
$$

The eigenvalues of the matrix $A$ are negative if, and only if,

For matrix B, we obtain

$$
\alpha_{1} \mu_{1 S}^{\prime}>\mu_{1 X}^{\prime}
$$

$$
\begin{gathered}
\operatorname{tr}(B)=-D-\alpha_{5} X_{2}^{*} \mu_{2 S}^{\prime}+\mu_{2 X}^{\prime} X_{2}^{*} \\
\operatorname{det}(B)=X_{2}^{*} D\left(-\mu_{2 X}+\alpha_{5} \mu_{2 S}\right)
\end{gathered}
$$

The eigenvalues of the matrix $B$ are negative if, and only if,

$$
\alpha_{5} \mu_{2 S}^{\prime}>\mu_{2 X}^{\prime}
$$

As in the case of equilibrium $E_{5}^{i}$, the conditions of stability of the matrix C obtained at the equilibria $E_{7}^{i}$ cannot be easily deduced using the Routh-Hurwitz criterion in view of the complicated form of the characteristic polynomial presented as below: 
Table B1: Analytical study of the stability of MAD equilibria.

\begin{tabular}{|c|c|c|c|c|c|c|}
\hline Equilibria & Area & $D, S_{i n}$ & Trace of matrices & Determinant of matrices & Routh-Hurwitz criterion/condition of existence & nature of equilibrium \\
\hline \multirow[t]{14}{*}{$E_{1}$} & \multirow[t]{2}{*}{$\mathcal{J}_{0}$} & $D=0.35$ & $\operatorname{tr}(A)<0$ & $\operatorname{det}(A)>0$ & \multirow[t]{2}{*}{ all the coefficients of the first Routh column $>0$} & \multirow[t]{2}{*}{$E_{1}$ stable } \\
\hline & & $S_{i n}=6$ & $\operatorname{tr}(B)<0$ & $\operatorname{det}(B)>0$ & & \\
\hline & \multirow{2}{*}{$\mathcal{J}_{1}$} & $D=0.20$ & $\operatorname{tr}(A)<0$ & $\operatorname{det}(A)<0$ & \multirow[t]{2}{*}{ all the coefficients of the first Routh column $>0$} & \multirow[t]{2}{*}{$E_{1}$ unstable } \\
\hline & & $S_{\text {in }}=6$ & $\operatorname{tr}(B)<0$ & $\operatorname{det}(B)>0$ & & \\
\hline & \multirow[t]{2}{*}{$\mathcal{J}_{2}$} & $D=0.1$ & $\operatorname{tr}(A)>0$ & $\operatorname{det}(A)<0$ & \multirow[t]{2}{*}{ all the coefficients of the first Routh column $>0$} & \multirow[t]{2}{*}{$E_{1}$ unstable } \\
\hline & & $S_{\text {in }}=25$ & $\operatorname{tr}(B)<0$ & $\operatorname{det}(B)>0$ & & \\
\hline & \multirow{2}{*}{$\mathcal{J}_{3}$} & $D=0.07$ & $\operatorname{tr}(A)>0$ & $\operatorname{det}(A)<0$ & \multirow[t]{2}{*}{ all the coefficients of the first Routh column $>0$} & \\
\hline & & $S_{i n}=6$ & $\operatorname{tr}(B)<0$ & $\operatorname{det}(B)>0$ & & \\
\hline & \multirow[t]{2}{*}{$\mathcal{J}_{4}$} & $D=0.03$ & $\operatorname{tr}(A)>0$ & $\operatorname{det}(A)<0$ & \multirow[t]{2}{*}{ all the coefficients of the first Routh column $>0$} & \\
\hline & & $S_{\text {in }}=6$ & $\operatorname{tr}(B)<0$ & $\operatorname{det}(B)<0$ & & \\
\hline & \multirow[t]{2}{*}{$\mathcal{J}_{5}$} & $D=0.04$ & $\operatorname{tr}(A)>0$ & $\operatorname{det}(A)<0$ & \multirow[t]{2}{*}{ all the coefficients of the first Routh column $>0$} & \\
\hline & & $S_{\text {in }}=0.01$ & $\operatorname{tr}(B)<0$ & $\operatorname{det}(B)<0$ & & \\
\hline & \multirow[t]{2}{*}{$\mathcal{J}_{6}$} & $D=0.04$ & $\operatorname{tr}(A)>0$ & $\operatorname{det}(A)<0$ & \multirow[t]{2}{*}{ all the coefficients of the first Routh column $>0$} & able \\
\hline & & $S_{\text {in }}=29$ & $\operatorname{tr}(B)<0$ & $\operatorname{det}(B)<0$ & & \\
\hline \multirow[t]{12}{*}{$E_{4}$} & \multirow[t]{2}{*}{$\mathcal{J}_{1}$} & $D=0.15$ & $\operatorname{tr}(A)<0$ & $\operatorname{det}(A)>0$ & \multirow{6}{*}{$\begin{array}{l}\text { all the coefficients of the first Routh column }>0 \\
\text { all the coefficients of the first Routh column }>0 \text {, } \\
\text { not all the coefficients of the first Routh column }>0\end{array}$} & $E_{4}$ stable \\
\hline & & $S_{\text {in }}=6$ & $\operatorname{tr}(B)<0$ & $\operatorname{det}(B)>0$ & & \\
\hline & \multirow[t]{2}{*}{$\mathcal{J}_{2}$} & $D=0.1$ & $\operatorname{tr}(A)<0$ & $\operatorname{det}(A)>0$ & & \multirow[t]{2}{*}{$E_{4}$ stable } \\
\hline & & $S_{\text {in }}=25$ & $\operatorname{tr}(B)<0$ & $\operatorname{det}(B)>0$ & & \\
\hline & \multirow[t]{2}{*}{$\mathcal{J}_{3}$} & $D=0.07$ & $\operatorname{tr}(A)<0$ & $\operatorname{det}(A)>0$ & & $E_{4}$ unstable \\
\hline & & $S_{i n}=6$ & $\operatorname{tr}(B)<0$ & $\operatorname{det}(B)>0$ & & \\
\hline & $\mathcal{J}_{4}$ & $D=0.03$ & $\operatorname{tr}(A)<0$ & $\operatorname{det}(A)>0$ & not all the coefficients of the first Routh column $>0$ & $E_{4}$ unstable \\
\hline & & $S_{\text {in }}=6$ & $\operatorname{tr}(B)<0$ & $\operatorname{det}(B)<0$ & & \\
\hline & $\mathcal{J}_{5}$ & $D=0.04$ & $\operatorname{tr}(A)<0$ & $\operatorname{det}(A)>0$ & t Routh column $>0$ & $E_{4}$ unstable \\
\hline & & $S_{\text {in }}=0.01$ & $\operatorname{tr}(B)<0$ & $\operatorname{det}(B)<0$ & & \\
\hline & $\mathcal{J}_{6}$ & $D=0.04$ & $\operatorname{tr}(A)<0$ & $\operatorname{det}(A)>0$ & ats of the first Routh column $>0$ & $E_{4}$ unstable \\
\hline & & $S_{\text {in }}=29$ & $\operatorname{tr}(B)>0$ & $\operatorname{det}(B)<0$ & & \\
\hline$E_{5}^{1}$ & $\mathcal{J}_{2}$ & $D=0.1$ & $\operatorname{tr}(A)<0$ & $\operatorname{det}(A)>0$ & all the coefficients of the first Routh column $>0$ & $E_{5}^{1}$ stable \\
\hline & & $S_{\text {in }}=25$ & $\operatorname{tr}(B)<0$ & $\operatorname{det}(B)>0$ & & \\
\hline & $\mathcal{J}_{3}$ & $D=0.07$ & $\operatorname{tr}(A)<0$ & $\operatorname{det}(A)>0$ & all the coefficients of the first Routh column $>0$ & $E_{5}^{1}$ stable \\
\hline & & $S_{\text {in }}=6$ & $\operatorname{tr}(B)<0$ & $\operatorname{det}(B)>0$ & & \\
\hline & $\mathcal{J}_{4}$ & $D=0.03$ & $\operatorname{tr}(A)<0$ & $\operatorname{det}(A)>0$ & Cients of the first Routh column $>0$ & $E_{5}^{1}$ unstable \\
\hline & & $S_{i n}=6$ & $\operatorname{tr}(B)<0$ & $\operatorname{det}(B)<0$ & & \\
\hline & $\mathcal{J}_{6}$ & $D=0.04$ & $\operatorname{tr}(A)<0$ & $\operatorname{det}(A)>0$ & all the coefficients of the first Routh column $>0$ & $E_{5}^{1}$ unstable \\
\hline & & $S_{i n}=29$ & $\operatorname{tr}(B)<0$ & $\operatorname{det}(B)<0$ & & \\
\hline$E_{6}$ & $\mathcal{J}_{4}$ & $D=0.03$ & $\operatorname{tr}(A)<0$ & $\operatorname{det}(A)>0$ & not all the coefficients of the first Routh column $>0$ & $E_{6}$ unstable \\
\hline & & $S_{\text {in }}=6$ & $\operatorname{tr}(B)<0$ & $\operatorname{det}(B)>0$ & & \\
\hline & $\mathcal{J}_{5}$ & $D=0.04$ & $\operatorname{tr}(A)<0$ & $\operatorname{det}(A)>0$ & all the coefficients of the first Routh column $>0$ & $E_{6}$ stable \\
\hline & & $S_{\text {in }}=0.01$ & $\operatorname{tr}(B)<0$ & $\operatorname{det}(B)>0$ & & \\
\hline$E_{7}^{1}$ & $\mathcal{J}_{4}$ & $D=0.03$ & $\operatorname{tr}(A)<0$ & $\operatorname{det}(A)>0$ & all the coefficients of the first Routh column $>0$ & $E_{7}^{1}$ stable \\
\hline & & $S_{\text {in }}=6$ & $\operatorname{tr}(B)<0$ & & & \\
\hline & $\mathcal{J}_{6}$ & $D=0.04$ & $\operatorname{tr}(A)<0$ & & all the coefficients of the first Routh column $>0$ & $E_{7}^{1}$ stable \\
\hline & & $S_{\text {in }}=29$ & $\operatorname{tr}(B)<0$ & & & \\
\hline
\end{tabular}

$$
\begin{aligned}
& P_{E_{7}^{i}}=\Lambda^{3}+\frac{1}{\alpha_{9}}\left(\mu_{3 S}^{\prime} S_{3 i n}^{*} \alpha_{9}-\mu_{3 S}^{\prime} S_{3}^{*} \alpha_{9}-\alpha_{10} S_{3}^{*} \mu_{3 N}^{\prime}+2 \alpha_{9} D+\alpha_{10} \mu_{3 N}^{\prime} S_{3 i n}^{*}\right) \Lambda^{2}+\frac{1}{\alpha_{9}}(2 D \\
& \left.\mu_{3 S}^{\prime} S_{3 i n}^{*} \alpha_{9}+2 S_{3 i n}^{*} \alpha_{10} D \mu_{3 N}^{\prime}-2 S_{3}^{*} \alpha_{10} D \mu_{3 N}^{\prime}+\alpha_{9} D^{2}-2 S_{3}^{*} \alpha_{9} \mu_{3 S}^{\prime} D\right) \Lambda+\frac{1}{\alpha_{9}}\left(D^{2} \mu_{3 N}^{\prime}\right. \\
& \left.\alpha_{10} S_{3 i n}^{*}-S_{3}^{*} \alpha_{10} D^{2} \mu_{3 N}^{\prime}+S_{3 i n}^{*} \alpha_{9} D^{2} \mu_{3 S}^{\prime}-\mu_{3 S}^{\prime} D^{2} \alpha_{9} S_{3}^{*}\right)
\end{aligned}
$$

Notice that the Jacobian matrix was not evaluated around the equilibria $E_{2}$ and $E_{3}^{i}$ because they have never been stable (see Tables 3, 4, 5).

\section{Application}

The stability of Jacobian matrix is investigated for some values of $D$ and $S_{i n}$, using the previous analytical calculations as illustrated in Table B1. 


\section{Appendix C. Algorithm of the Operating Diagram}

The operating diagram is the two-parameter bifurcation scheme that shows how the system behaves when we vary two control parameters [28].

Due to the higher order of the MAD model, the system's behavior with respect to the changes of $D, S_{i n}$ and $N_{i n}$ cannot be investigated analytically. Hence, we obtain the operating diagram of the MAD model using numerical simulations realized with MATLAB.

Initially the script performs a calculation of the eigenvalues of the Jacobian matrix defined for the MAD model. These calculations are evaluated for each equilibrium of the MAD around certain values of $D$ and $S_{i n}$. According to the sign of the eigenvalues and the conditions of existence, we assign to each of 10 equilibria: stability, instability or non existence. This procedure gives a set of combinations matching pairs of values $\left(D, S_{i n}\right)$.

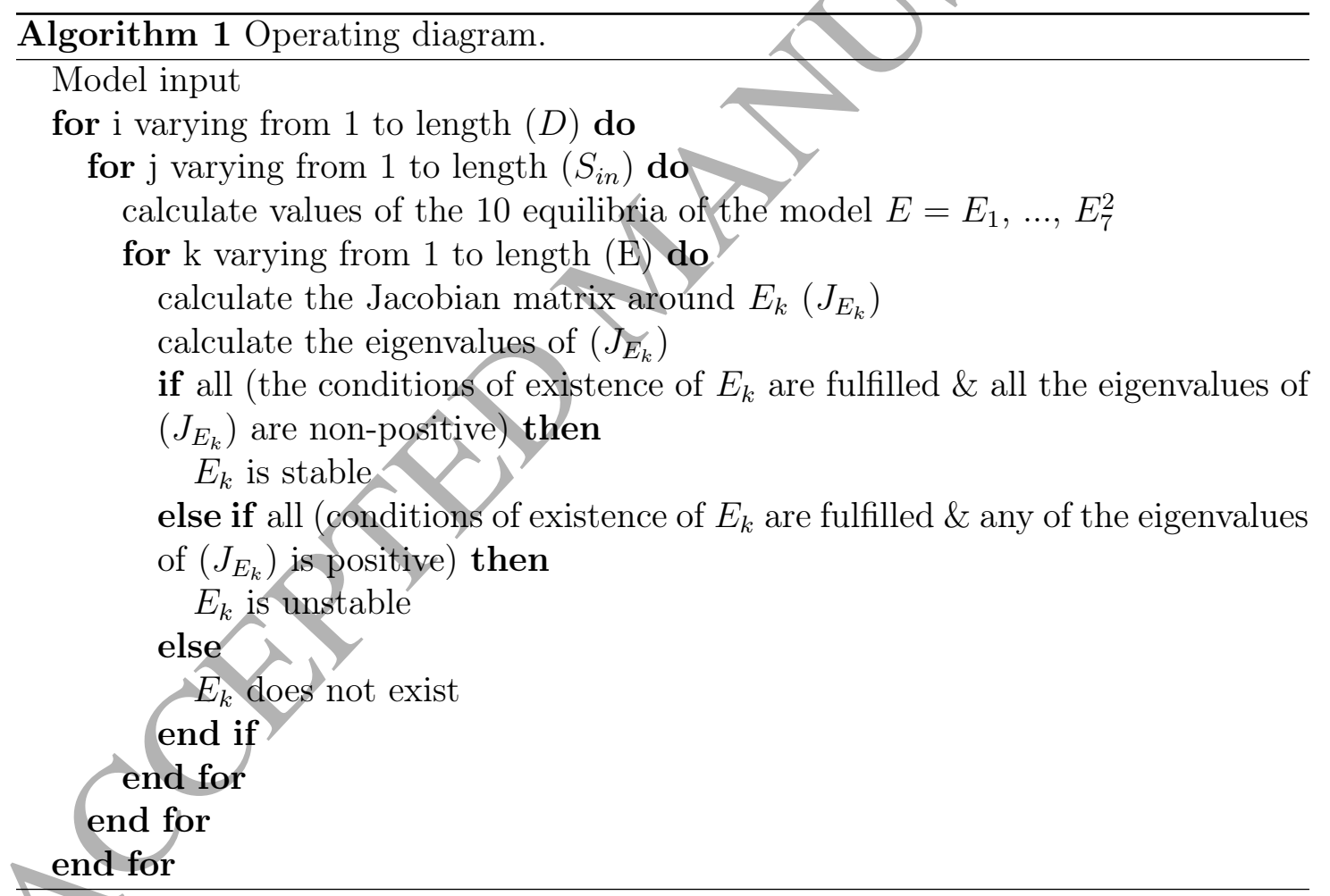




\section{Acknowledgements}

Authors thank the Hubert Curien Tassili project 15MDU949 and the EuroMediterranean TREASURE Research Network (cf. www.inra.fr/treasure) for their financial support. Authors also thank Jean-Philippe Steyer, Francis Mairet and Olivier Bernard for fruitful discussions about this work.

\section{References}

\section{References}

[1] U. Baserga, Co-digestion in agricultural biogas plants. Digestion of organic residues and energy grass for biogas production, J. Food and Agriculture Organization of the United Nation. 512 (1998).

[2] E. Kovács, R. Wirth, G. Maróti, Z. Bagi, K. Nagł J. Minárovits, G. Rákhely, K.L. Kovács, Augmented biogas production from protein-rich substrates and associated metagenomic changes, J. Bioresource Technology. 178 (2015) 254261.

[3] O. Yenigün, B. Demirel, Ammonia inhibition in anaerobic digestion: A review, J. Process Biochemistry. 48 (2013) 901-911.

[4] F. Lü, M. Chen, P-J. He, L-M. Shao, Effects of Ammonia on Acidogenesis of Protein-Rich Organic Wastes, J. Environmental Engineering Science. 25 (2008) 114-122.

[5] F. Omil, R. Méndez, J.M. Lema, Anaerobic treatment of saline wastewater under high sulphide and ammonia content, J. Bioresource Technology. 54 (1995) 268278 .

[6] L. Guerrero, F Omil, R. Méndez, J.M. Lema, Anaerobic hydrolysis and acidogenesis of wastewaters from food industries with high content of organic solids and protein, J. Water Research. 33 (1999) 3281-3290.

[7] L. Zhang, D. Jahng, Long-term anaerobic digestion of food waste stabilized by trace elements, J. Waste Management. 32 (2012) 1509-1515.

[8] C, Resch, A. Wörl, R. Waltenberger, R. Braun, R. Kirchmayr, Enhancement options for the utilisation of nitrogen rich animal by-products in anaerobic digestion, J. Bioresource Technology. 102 (2011) 2503-2510. 
[9] B. Zhang, L. Zhang, S. Zhang, H. Shi, W. Cai, The influence of pH on hydrolysis and acidogenesis of kitchen wastes in two-phase anaerobic digestion, J. Environ. Technol. 26 (2005) 329-340.

[10] M. Ortner, K. Leitzinger, S. Skupien, G. Bochmann, W. Fuchs, Efficient anaerobic mono-digestion of N-rich slaughterhouse waste: influence of ammonia, temperature and trace elements, J. Bioresour. Technol. 174 (2014) 222-232.

[11] A. Hejnfelt, I. Angelidaki, Anaerobic digestion of slaughterhouse by-products, J. Biomass. Bioenergy. 33 (2009) 1046-1054.

[12] A.O. Wagner, P. Lins, C. Malin, C. Reitschuler, P. Illmer, Ampact of protein-, lipid- and cellulose-containing complex substrates on biogas Production and microbial communities in batch experiment, J. Science of the Total Environment. 458-460, 256-266 (2013).

[13] D.J. Batstone, J. Keller, R.B. Newell, M. Newlánd, Modelling anaerobic degradation of complex wastewater. I: model development, J. Bioresource Technology. 75 (2013) 67-74.

[14] LY. Lokshina, V. Vavilin, E. Salminen, J. Rintála, Modeling of anaerobic degradation of solid slaughterhouse waste: inhibition effects of long-chain fatty acids or ammonia, J. Appl. Biochem. Biotechnol. 109 (2003) 15-32.

[15] I. Angelidaki, B. K. Ahring, Methods for increasing the biogas potential from the recalcitrant organic matter contained in manure In: Mata-Alvarez, J., Tilche, A., Cecchi, F. (Eds.), Proceedings of the Second International Symposium on Anaerobic Digestion of Solid Wastes, Barcelona, 1999.

[16] F. Mairet, O. Bernard, E. Cameron, M. Ras, L. Lardon, J-P. Steyer, B. Chachuat, Three-Reaction Model for the Anaerobic Digestion of Microalgae, J. Biotechnology and Bioengineering. 109 (2011) 415-429.

[17] F. Mairet, O Bernard, M. Ras, L. Lardon, J-P. Steyer, Modeling anaerobic digestion of microalgae using ADM1, J. Bioresource Technology. 102 (2011) 6823-6829.

[18] M.J Wade, J. Oakley, S. Harbisher, N.G. Parker, J. Dolfing, MI-Sim: A MATLAB package for the numerical analysis of microbial ecological interactions, J. Public Library of Science (PLoS ONE). 12 (2017) 1-15. 
[19] M.J. Wade, R.W. Pattinson, N.G. Parker, J. Dolfing, Emergent behaviour in a chlorophenol-mineralising three-tiered microbial 'food web', J. Theoretical Biology. 389 (2016) 171-186.

[20] B. K. Ahring, M. Sandberg, I. Angelidaki, Volatile fatty acids as indicators of process imbalance in anaerobic digestors, J. Appl. Microbiol. Biotechnol. 43 (1994) 559-565.

[21] D.J. Batstone, J. Keller, I. Angelidaki, S.V. Kalyuzhnyi, S.G. Pavlostathis, A. Rozzi, W.T. Sanders, H. Siegrist, V.A. Vavilin, Anaerobic digestion Model No.1(ADM1), J. Water. Sci. Techno. 45 (2002) 65-73.

[22] O. Bernard, Z. Hadj-Sadock, D. Dochain, A. Genovesi, J-P. Steyer, Dynamical Model Development and Parameter Identification for an Anaerobic Wastewater Treatment Process, J. Biotechnologie and Bioengineering. 75 (2001) 424-438.

[23] B. Benyahia, T. Sari, B. Cherki, J. Harmand, Bifurcation and stability analysis of a two step model for monitoring anaerobic digestion processes, J. Process Control. 22 (2012) 1008-1019.

[24] A. Bornhöft, R. Hanke-Rauschenbach, K. Sumdmacher, Steady-state analysis of the Anaerobic Digestion Model No. 1 (ADM1), J. Nonlinear Dynamics. 73 (2013) 535-549.

[25] R. Fekih-Salem, N. Abdellatif, T. Sari, J. Harmand, Analyse mathématique d'un modèle de digestion anaérobie à trois étapes, J. ARIMA. 17 (2014) 53-71.

[26] Z. Khedim, B. Benyahia, F. Mairet, J-P. Steyer, J. Harmand, Reduction of a complex biotechnological process model using state-variable association methodApplication to the anaerobic digestion of micro-algae, Proceedings of the 2017 International Conference on Control, Automation and Diagnosis, Hammamet, 2017.

[27] S. Hassam, E. Ficara, A. Leva, J. Harmand, A generic and systematic procedure to derive a simplified model from the Anaerobic Digestion Model No.1 (ADM1), J. Biochemical Engineering. 99 (2015) 193-203.

[28] T. Sari, M. J. Wade, Generalised approach to modelling a three-tiered microbial food-web, J. Mathematical Biosciences. 291 (2017) 21-37. 